DIVISION OF THE HUMANITIES AND SOCIAL SCIENCES

CALIFORNIA INSTITUTE OF TECHNOLOGY

PASADENA, CALIFORNIA 91125

EQUILIBRIUM AGENDA FORMATION

Bhaskar Dutta

University of Warwick

Matthew O. Jackson

California Institute of Technolgy

Michele Le Breton

GREMAQ and IDEI

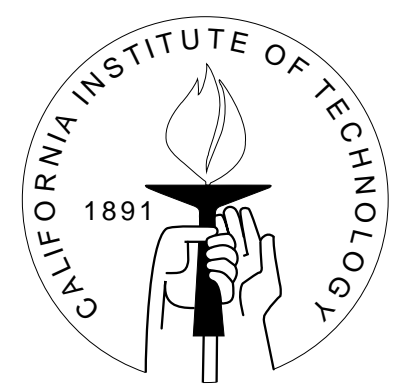

SOCIAL SCIENCE WORKING PAPER 1152

November 2002 


\title{
Equilibrium Agenda Formation
}

\author{
Bhaskar Dutta Matthew O. Jackson Michele Le Breton
}

\begin{abstract}
We develop a definition of equilibrium for agenda formation in general voting settings. The definition is independent of any protocol. We show that the set of equilibrium outcomes for any Pareto efficient voting rule is uniquely determined, and in fact coincides with that of the outcomes generated by considering all full agendas. Under voting by successive elimination (or amendment), the set of equilibrium outcomes corresponds with the Banks set. We also examine the implications in several specific settings and show that studying equilibrium agendas can lead to sharp predictions, in contrast with well-known "chaos" theorems.
\end{abstract}

JEL classification numbers: D71, D72

Key words: agenda formation, voting, endogenous agendas, equilibrium 


\title{
Equilibrium Agenda Formation*
}

\author{
Bhaskar Dutta \\ Matthew O. Jackson \\ Michele Le Breton
}

\section{Introduction}

The importance of agenda formation in a wide variety of settings, ranging from committees to popular elections, is self-evident. In fact, in some legislative settings where the voting on specific bills is highly predictable, one might argue that the most interesting strategic interaction takes place in the formation of the agenda.

Indeed, the wide literature that analyzes various aspects of voting recognizes the importance of the agenda, and has shown how important it can be (e.g., McKelvey $(1976,1979))$. Nevertheless, we still lack tractable models of agenda formation, and a detailed understanding of how the formation of the agenda ultimately affects the outcome of voting. To quote Ordeshook (1993):

More problematic is the issue of endogenous agendas, the process whereby agendas are formed via the sequential introduction and labeling of alternatives to be voted on. ... The particular problem is that to apply game theory we must provide a game form that specifies precisely the identity of decision makers, the sequence with which they make decisions, and the information at their disposal when they act. And although agenda voting, like simple descriptions of elections, lends itself readily to the construction of such form, the processes whereby agendas are formed is far less structured and, thereby, less amenable to unambiguous game-theoretical analysis.

Ordeshook's statement points out the difficulty of modeling agenda formation stemming from the lack of a clearly defined game form.

In this paper we provide a model of agenda formation, and in particular one that does not rest on a specific game form or protocol. Nevertheless, we still wish to capture strategic reasoning. The way in which we do this is to use a backwards induction approach. We examine the continuation equilibria that might extend from any given agenda, based

${ }^{*}$ Financial support under NSF grant SES-9986190 and an RTDF grant from the University of Warwick are gratefully acknowledged. 
on previous inductions where equilibrium continuations have been defined starting from longer agendas.

The key to how we are able to make progress in defining equilibrium agenda formation without reference to a specific protocol is through a simple observation that ends up having powerful implications. That observation concerns when it is possible to stop at some agenda under an equilibrium: It is an equilibrium to stop at some agenda only if no agent prefers any continuation equilibrium. We show that the sequential rationality and stopping conditions alone provide strong conclusions for what the set of equilibrium agendas can include.

In particular, we show that if a voting rule always selects an outcome that is Pareto efficient relative to the agenda that has been proposed, then sequential rationality and stopping conditions imply that equilibrium agendas will result in voting outcomes that are Pareto efficient overall. Moreover, one of our main results states that for Pareto efficient voting rules the equilibrium outcomes will always be a subset of what might arise from considering the set of complete agendas (including all outcomes). This result turns out to allow us to make fairly sharp predictions concerning equilibrium agendas in many settings. For example, if the voting rule does not depend on the specific order of the agenda, then equilibrium agendas result in a unique outcome which is that when all alternatives are included in the voting. This also has important implications for voting rules where the order of the agenda does matter, such as the well-studied example of voting by successive elimination. There we show that equilibrium agendas always result in outcomes that lie in the Banks' set. Similarly, for voting rules that always pick outcomes that lie in the top cycle of the alternatives on the agenda, we show that the equilibrium agendas must result in outcomes that lie in the top-cycle of all alternatives. So, if for instance, a Condorcet winner exists and the voting rule is Condorcet consistent, then all equilibrium agendas include (and thus result in) the Condorcet winner.

While sequential rationality and stopping conditions already have a substantial impact on identifying agendas, we can impose further conditions to produce more specific equilibrium sets and predictions. We also examine a consistency condition which requires that if one continuation is an equilibrium, and some agent prefers another continuation (which would be an equilibrium if the agenda is extended by the addition of one alternative), then this second continuation must also be an equilibrium. The converse is also imposed: unless there is a unique equilibrium agenda, all equilibrium agendas must be rationalizable in that at least some agent must weakly prefer them to some other equilibrium continuation. In the context of Pareto efficient voting rules, we show that the consistency condition ties down the set of equilibrium agendas uniquely and provides a simple algorithm for identifying them.

\section{Some Related Literature}

Part of the motivation behind our analysis comes from the literature on "chaos" theorems. For instance, McKelvey $(1976,1979)$ has shown that in the context of majority 
rule and Euclidean settings, the top cycle of alternatives is either a singleton (a Condorcet winner) or the whole space. And, as Plott (1973) has shown, the second case is the generic one. ${ }^{1}$ This implies that in most cases, starting from one alternative one can find a sequence of alternatives leading to any other, where each one in the sequence beats the previous one under a (myopic) majority vote. While the conclusion that one should draw from such a result and whether or not "chaos" is an appropriate nickname has been debated, it is clear that such a result makes it critical to have an understanding of equilibrium agendas - otherwise one can be left without any prediction. This is essentially the primary motivation for our analysis. As such, we come back below to examine the predictions our equilibrium notion makes in the context of voting by successive elimination, and discuss the relation to chaos theorems.

An alternative approach to modeling agenda formation is to assume a specific protocol, for instance a completely specified extensive form game where each possible move of every player is explicit, and analyze its implications. For instance random recognition rules were studied in the context of multilateral bargaining (divide-the- dollar games) by Baron and Ferejohn (1989) and a literature that followed. That approach provides for strong analytical conclusions. However, that approach is not so tractable outside of the distributive setting as the games become quite difficult to analyze. Moreover, there are many applications where the protocol is not clear, as the above quote of Ordeshook points out. The advantages to the approach taken in this paper are that it can be applied to a general class of voting problems, where for instance, Euclidean preferences may not be appropriate; and it makes protocol-free predictions.

With regard to making protocol-free predictions, we remark that the sets of equilibria uncovered here should be viewed as a set of potential equilibria. Adding more knowledge of the specific protocol may induce selections from the set we identify, and result in more specific predictions. Nevertheless, as we shall show, fairly minimal requirements on the equilibrium set already allow for some tight predictions in the context of a variety of voting rules. Thus there are important aspects of equilibrium agendas that can be characterized without detailed knowledge of the protocol.

Work on equilibrium agenda formation has also been done in other contexts. For example, Banks and Gasmi (1987) examined equilibrium agenda formation in three person committees. Their analysis is of a Euclidean setting and one where the three committee members can make only one proposal each, and so agendas are truncated. Specifying the problem to this level leads to sharp predictions. More recently, Penn (2001), in the context of three person divide-the-dollar games has extended the analysis to allow for arbitrary agenda lengths by a clever adaptation to infinite agendas, and shows that sharp predictions again result (but differ from those of Banks and Gasmi). The above results are very encouraging in the face of "chaos" theorems, and may be thought of as answering those theorems by saying that if we do model agenda formation, then we can make specific predictions. Nevertheless, the above analyses come in very specific settings and

\footnotetext{
${ }^{1}$ See Austen-Smith and Banks (1999) for a nice discussion of this literature and extensions of McKelvey's theorem.
} 
are dependent upon the geometry of Euclidean preferences, and in some cases having three proposers and having a strong symmetry among them. Our analysis attempts to provide an equilibrium definition that can be applied to a more general set of problems. Our main motivation is to develop a concept that does not require such specific geometry, and at the same time does not demand detailed specification of the proposal protocol. ${ }^{2}$ As such, the predictions our analysis makes are not always as crisp; but nevertheless are fairly specific in many settings.

Equilibrium agenda formation has also been analyzed in the setting of strategiccandidacy. For instance, in Osborne and Slivinski (1996) and Besley and Coate's (1997) models of citizen- candidates the decision to enter an election and take a position is studied under equilibrium. In other work (Dutta, Jackson, and Le Breton (2001, 2002)) we have examined the properties of equilibrium sets of candidates for a variety of voting rules and for voting by successive elimination. Part of our motivation in studying agenda formation more broadly comes from noting the impact of strategic candidacy. As shown in Dutta, Jackson, and Le Breton (2001), all non-dictatorial voting rules result in some situations where some non-winning candidates have an incentive to exit the election to manipulate the outcome. ${ }^{3}$ This implies that strategic agenda formation is critical to all voting rules, at least when the agenda consists of candidates. Moreover, Dutta, Jackson, and Le Breton $(2001,2002)$ provide examples where the equilibrium candidate entry decisions result in Pareto inefficient outcomes even though the voting procedure is Pareto efficient. While the issue of strategic candidacy is an important example of endogenous agenda formation, modeling agenda formation more generally requires a different approach. Most importantly, the candidacy decision ultimately rests with the candidate. ${ }^{4}$ This means that the proposal abilities of agents are limited. This provides for different strategic considerations than, for instance, in a legislative setting where proposers are not restricted in the alternatives that they may propose. One important question (that we answer here in the affirmative) is whether or not such problems of inefficient outcomes would be overcome in settings in which any voter can propose any alternative. When we compare the outcomes of strategic agenda formation in the context of strategic candidacy and in the more general setting where proposers are not limited; we see that there are important distinctions.

Another branch of the literature that has touched on equilibrium agenda formation is that which has looked at sophisticated voting by successive elimination. In particular, a definition of equilibrium agendas appears in work by Miller, Grofman, and Feld (1990). In their analysis an agenda is an equilibrium if nobody would gain by adding some alternative to the current agenda. The important differences between such a definition and the ones presented here are in the beliefs of the proposers. The definition of Miller,

\footnotetext{
${ }^{2}$ Another distinction is that our approach is based on one of inductively defining equilibrium continuations, and so equilibria are defined in a manner that can be thought of as analogous to subgame perfect equilibrium (but without a game form). Instead the Banks and Gasmi (1987) and Penn (2001) formulations use a "maximin-Stackelberg" based equilibrium notion.

${ }^{3}$ See also Eraslan and McLennan (2000), Rodriguez (2000) and Ehlers and Weymark (2001) for further results and consideration of multi-valued voting rules.

${ }^{4}$ Even if one allows candidates to be nominated, they usually have the option to decline to run.
} 
Grofman, and Feld does not account for the fact that in many cases the agenda will not end, but instead will be subject to further modifications. Thus, proposers are acting myopically. ${ }^{5}$ If proposers can make any predictions about continuations, rather than myopically assuming the agenda will end, then the outcome in general will be quite different. This emphasizes an important aspect of our definitions. Incorporating such sequential rationality and anticipating equilibrium continuations is the foundation on which we build our definitions. We come back to examine the impact of this feature below, when we apply our definitions to voting by successive elimination.

Finally, we mention a distantly related literature in terms of applications and specifics; but more closely related in terms of finding equilibrium definitions that are not tied down to protocol specification. In particular, the literature on coalition formation (and on coalitional bargaining) faces a similar difficulty to that expressed in the quote of Ordeshook above. Writing down specific bargaining protocols allows for sharp predictions, but ones that may not be robust and are not so easily adapted to settings where the protocol is not obvious. Chwe (1994) provides a definition of consistent sets of alternatives that might come out of coalitional bargaining settings, that is not dependent on any specific protocol and yet still makes intuitively appealing predictions in many applications (see also Greenberg (1990) for such an analysis of general social situations). Our approach here is intended to do the same thing for agenda formation problems. While there is a parallel in spirit, the actual equilibrium definition that we provide and the issues we face are more specific to agenda formation and bear little resemblance to that in Chwe's (or Greenberg's) work. ${ }^{6}$

\section{Definitions}

\section{Alternatives}

There is a set of alternatives $X$. Generic elements are denoted $x, y$, and $z$.

We begin the analysis with the case where $X$ is finite and with $\# X=m$, as this brings out the intuitions most clearly. We then return to show how our analysis extends to the infinite case in Section 5.7.

Society will select one of these alternatives. These may be potential bills that a legislature might enact, a set of candidates that a society might elect, or a list of potential decisions that a committee might reach.

\footnotetext{
${ }^{5}$ Austen-Smith (1987), Duggan (2002) and Groseclose and Krehbiel (1993) also examine equilibrium agenda formation under voting by successive elimination. Their approach does not have the myopic problem of Miller, Grofman, and Feld; which they avoid by assuming a fixed ordering (or lottery) over individuals who can each make a single proposal. However, their focus is on analyzing (sophisticated sincerity) under the amendment procedure, and not on characterizing equilibrium agendas more broadly.

${ }^{6}$ Let us also point out that our use of the word consistency has no relationship to that of Chwe's consistent sets.
} 


\section{Voters or Decision Makers}

The set $N=\{1, \ldots, n\}$ is a finite set of voters.

These are the individuals who are involved in determining the agenda and the outcome from that agenda. In Section 6 we discuss the possibility of having special roles for some individuals.

\section{Preferences}

Individuals have preferences over the set of alternatives represented by a complete and transitive binary relation, $R_{i}$. The strict preference relation associated with $R_{i}$ is denoted $P_{i}$, and is defined by $x P_{i} y$ if and only if not $y R_{i} x$. As usual, knowing $P_{i}$ similarly defines $R_{i}$, and so we keep track of the strict relationship with the weak one being inferred.

Let $\mathcal{P}$ denote the set of admissible profiles of preference relations. The notation $P \in \mathcal{P}$ denotes a generic profile $P=\left(P_{1}, \ldots, P_{n}\right)$.

In some applications $\mathcal{P}$ will be a restricted domain. A number of different examples appear in what follows.

\section{Agendas}

An agenda of length $k \in\{1, \ldots, m\}$ is a finite vector of alternatives $\left(x_{1}, \ldots, x_{k}\right) \in X^{k}$, with the restriction that $x_{i} \neq x_{j}$ for each $i \neq j$.

Let $A^{k}$ denote the set of agendas of length $k$, and let $A=\cup_{k=1}^{m} A^{k}$ be the set of all agendas.

The restriction that the same alternative not appear more than once in an agenda is common to many legislative and committee settings. Given that the set of alternatives $X$ could be quite large and dense, this does not prevent an alternative and a close approximation of it from appearing in an agenda.

Let us emphasize that depending on how the voting procedure works, the sequence of the agenda may or may not matter. For instance if the agenda is simply a list of nominated candidates and some neutral voting procedure is used, then the agendas $(x, y, z)$ and $(z, y, x)$ would be equivalent. However, if the voting procedure is non-neutral, then the sequence can be important. For instance, under voting by successive elimination where proposed alternatives are voted upon in reverse order of their proposal the agendas $(x, y, z)$ and $(z, y, x)$ are not equivalent and could lead to different outcomes.

\section{Extensions of an Agenda}


In many situations of interest, some part of an agenda will already be on the table. For example, if there is a status quo, then it may take the first place in any agenda that follows. More generally, in building a definition of equilibrium we need to be able to make predictions starting from various existing agendas and so it is useful to consider the concept of the extensions of a given agenda.

With this in mind, for any $k$ and $a \in A^{k}$ let $A(a)$ to be the set of all agendas that agree with $a$ in the first $k$ spots. That is,

$$
A(a)=\left\{a^{\prime} \in A \mid a_{h}^{\prime}=a_{h} \forall h \in\{1, \ldots, k\}\right\} .
$$

We let $A^{\text {Full }}(a)=A(a) \cap A^{m}$ denote the agendas of full length that are extensions of $a$.

\section{Voting Procedures}

A voting procedure is a function $V: A \times \mathcal{P} \rightarrow X$ such that $V(a) \in a$ for all $a \in A$ and $P \in \mathcal{P}$.

A voting procedure thus summarizes the choice the society would make from a given agenda at a given preference profile.

Let us emphasize that this formulation is very flexible and allows for many applications. For instance, it could be that $V$ is determined by strategic voting or instead by sincere voting. Also, $V$ might depend on the ordering of the agenda or it might not; and $V$ might be anonymous, or it might treat some voters specially.

The details of how $V$ is determined will not be important in developing our definition of equilibrium agenda formation. Later, in providing some results about the properties of equilibria, we will specify some properties of potential voting rules $V$ and examine some specific voting rules.

The one restrictive assumption that is implicit in our specification of a voting rule is that it is single-valued. This may involve some deterministic tie-breaking rule in the event that some alternatives are tied. It may also be that under a given voting procedure there are multiple voting equilibria (taking the agenda as given), and hence multiple possible outcomes. In order to adapt our approach to situations where there may be several voting outcomes for a given agenda, one can apply our definitions to each possible equilibrium selection of the voting procedure. Although we do not do this explicitly here, it is an easy extension of our results.

In much of what follows, the set of alternatives $X$, the voting rule $V$, and the preference profile $P$ will be given, and so we omit their notation as arguments in various functions. 


\section{Equilibrium Agendas}

Before presenting the formal definitions of equilibrium, we begin with a simple example to motivate and illustrate the definitions.

Example 1

$X=\{x, y, z\}$ and $x$ is the status quo.

The voters' preferences form a classic cycle:

- $x P_{1} y P_{1} z$

- $y P_{2} z P_{2} x$

- $z P_{3} x P_{3} y$

Here $x$ beats $y, y$ beats $z$, and $z$ beats $x$ under majority rule.

The voting rule is sincere voting by successive elimination. For instance, if the agenda is $(x, z, y)$, then first a vote is held between $y$ and $z$, and then the winner is matched against $x$. Under sincere voting, the outcome of this agenda would be $x$, as $y$ would defeat $z$ and then $x$ would beat $y{ }^{7}$ Here, the only possible outcomes are $x$ from agendas $(x, y, z),(x, z, y),(x, y)$ and $x$; and $z$ from agenda $(x, z)$.

Let us discuss equilibrium conditions based on this example. Once an agenda of three alternatives has been reached, there are no alternatives left to propose, and so an equilibrium continuation is simply the agenda in question. Next let us step back and consider an agenda of length 2 that starts with the status quo $x$. There are only two such agendas to consider. One is the agenda $(x, z)$. If this agenda is reached, then agent 1 by adding the alternative $y$ would change the outcome from $z$ to $x$. This would make agent 1 better off, and so the agenda $(x, z)$ would not be stable to amendment. ${ }^{8}$ This suggests

\footnotetext{
${ }^{7}$ A situation which approximately fits this one is that of the Powell amendment discussed by Denzau, Riker, and Shepsle (1985) and others. The alternative $x$ would be the status quo of no U.S. federal funding of local public schools. The bill $z$ under consideration in the House of Representatives was one that would introduce some federal funding of local public schools. The amendment to the bill $y$ introduced by Powell was to deny federal funding to public schools that practiced segregation (this was in the 1950's). As Denzau, Riker and Shepsle argue, sincere voting could be explained by the difficulty in explaining voting against the Powell amendment to one's constituency. In fact, the situation had some mixture of sincere and sophisticated voting, as some representatives who opposed funding (and supported segregation) may have voted for the Powell amendment in the first round and then against it in the second round. So there may have been some conservative representatives who had the preferences of voter 1 except with $z$ and $y$ reversed, but who when voting strategically would vote the same as voter 1 would vote when voting sincerely. The motivations behind the voting are not so critical to the example. All that matters to the analysis is that from a full agenda the outcome would be $x$, while from an agenda of only $x$ and $z$ the outcome would be $z$.

${ }^{8}$ Interestingly, in this example if we require a second agent to support a proposal in order for it to become part of the agenda, neither of the remaining agents would second the proposal. This turns out to be an artifact of the sincere voting and also the fact that there is only one agent with any given preference profile. We discuss how this is not a problem for sophisticated voting below.
} 
one of the conditions in our equilibrium definition: that stopping at a given agenda is an equilibrium if and only if there is no agent who can benefit from advancing the agenda to some further continuation equilibrium. So, the only continuation equilibrium following $(x, z)$ is the agenda $(x, z, y)$. Next, let us back things up. Given the agenda $x$ in place, if some agent proposes $z$ next, then she should anticipate that the result will be the full agenda $(x, z, y)$ with outcome $x$. This embodies another part of the equilibrium definition: agents should anticipate equilibrium continuations from extensions of an agenda. In this case, no matter what happens after $x$, any continuation equilibrium must lead to the outcome of $x$. This actually means that stopping at $x$ can be an equilibrium. Whether or not the other agendas that lead to $x$ are also included as equilibrium continuations from $x$, is something that is not mandated by our basic definitions of equilibrium. However, a further consistency condition that we add would imply that the other agendas leading to $x$ would also be equilibria in this example. ${ }^{9}$

With some of the basic ideas from this simple example in hand, let us now consider the full definition of equilibrium agendas.

First, notice as in the above example, defining behavior at one agenda requires having some notion of what will happen following various extensions of the given agenda. Thus, the definition involves sets of continuation equilibria to be defined from each starting point. This is necessarily a set of sets, where a set of continuation equilibria is specified starting from each possible agenda.

We deliberately impose only weak requirements in defining equilibrium sets. Although taking such an approach allows for various collections to satisfy the definition, these weak requirements already have substantial implications for which outcomes might be reached.

A collection of sets of continuation equilibria is a collection $\{C E(a)\}_{a \in A}$, where $C E(a) \subset A(a)$ for each $a \in A$, that satisfies the following properties. ${ }^{10}$

Given $\{C E(a)\}_{a \in A}$, let

$$
C^{+}(a)=\cup_{x \notin a} C E((a, x)) .
$$

So $C^{+}(a)$ is the set of all continuation equilibria that could result if some alternative is added to an existing agenda $a .^{11}$

A collection of continuation equilibrium sets satisfies the following for each $a \in A$ :

\footnotetext{
${ }^{9}$ Interestingly, it would seem that Powell, who introduced the amendment $y$ to the agenda $(x, z)$ would have actually preferred the outcome of that agenda $(z)$ to the outcome of $x$ that resulted from the agenda $(x, z, y)$ due to the addition of his amendment $y$. However, given that that if Powell had not introduced the amendment $y$, then someone else would have liked to; Powell might as well have proposed the amendment, especially as it was one that he and much of his constituency strongly cared about.

${ }^{10}$ As mentioned above, we are taking $X, V$, and $P$ as given and omit their notation as arguments in $C E$. We will be explicit if these are varying.

${ }^{11}$ We remark that if $a \in A^{m}$, then $C^{+}(a)=\emptyset$. Under (CE1) and (CE2) below, this implies that $C E(a)=\{a\}$ if $a \in A^{m}$.
} 
(CE1) (Equilibrium Continuations) $C E(a)$ is a nonempty subset of $\{a\} \cup C^{+}(a)$ and

(CE2) (Stopping Requirements) $a \in C E(a)$ if and only if $V(a) R_{i} V\left(a^{\prime}\right)$ for all $a^{\prime} \in C^{+}(a)$ and for all $i \in N$.

Part (CE1) is a sequential rationality condition that simply says that the possibilities from any agenda $a$ are either to stop at $a$, or to add a new alternative to the agenda and then follow some continuation equilibrium from the resulting agenda. This is a condition that essentially just requires that the sets of equilibria for different agendas have some minimal relationship to each other: if agents anticipate that $a^{\prime}=(a, x, \ldots)$ is a continuation equilibrium starting at $a$, then they must also expect it to still be a continuation equilibrium when they have reached $(a, x)$.

Part (CE2) describes conditions under which it can be an equilibrium for agents to 'stop' at $a$. If every agent finds that $V(a)$ is at least as good as the outcome corresponding to any other possible continuation equilibrium, then no agent has an incentive to extend $a$. Conversely, if some agent $i$ finds the voting outcome corresponding to some continuation equilibrium strictly preferred to $V(a)$, then this $i$ will rather make a proposal and follow the preferred continuation equilibrium, and the agenda will not stop at $a$.

One of our main themes developed below is that these minimal conditions already have some very strong implications and imply a great deal about sets of equilibria.

While imposing some restrictions on collections of sets of continuation equilibria, conditions (CE1) and (CE2) can still allow for a multiplicity of collections of equilibrium continuations that satisfy the definition. Essentially, (CE1) and (CE2) give us some weak limitations on what can be in the set of equilibria, but they do not tell us much about which agendas must be included in the set. Consistency (CE3), below, addresses this issue.

We say that an agenda $a^{\prime}=(a, x, \ldots)$ is rationalizable (relative to $a$ ) if $a^{\prime} \in C^{+}(a)$ and there exists $i \in N$ and $a^{\prime \prime} \in C E(a)$ with either $a^{\prime \prime}=(a, y, \ldots)$ with $y \neq x$ or $a^{\prime \prime}=a$ such that $V\left(a^{\prime}\right) R_{i} V\left(a^{\prime \prime}\right)$.

The idea of rationalizability is that $i$ proposes adding $x$ to the agenda $a$ under the belief that it will result in the agenda $a^{\prime}$, and that if $i$ does not propose adding $x$ then instead the continuation would be $a^{\prime \prime}$. As $a^{\prime \prime}$ is a continuation equilibrium, this belief can be justified.

We say that a collection of sets of continuation equilibria is consistent if it satisfies

(CE3) (Consistency) If $a^{\prime} \in C^{+}(a)$ is rationalizable, then $a^{\prime} \in C E(a)$. Conversely, if $a^{\prime}=(a, x, \ldots) \in C E(a)$ and either $a \in C E(a)$ or $a^{\prime \prime}=(a, y, \ldots) \in C E(a)$ for some $y \neq x$, then $a^{\prime}$ is rationalizable. 
Part (CE3) is a consistency condition on the collections of sets of continuation equilibria. It says the equilibrium continuations are those which are rationalizable, subject to two exceptions. One is that stopping is handled under (CE2), and so the rationalization of $a$ itself is already addressed. The second is that an equilibrium continuation agenda does not need to be rationalizable if it is a "unique" equilibrium continuation. Note that in this second case, the first part of the condition implies that all agents unanimously find the outcomes under $(a, x, \ldots)$ preferred to stopping or adding any other alternative to $a$.

Later, we come back to discuss other notions of rationalizability and consistency.

We point out some important aspects of the above definitions.

First, the definitions necessarily involve a whole collection of $\{C E(a)\}$, one set for each $a \in A$. This reflects the forward-looking aspect of the definition. In order to know what is an equilibrium starting at one agenda, one has to be able to anticipate what will happen starting at extensions of that agenda. ${ }^{12}$

Second, there always exists at least one collection $\{C E(a)\}$ satisfying (CE1)-(CE3), which is easily seen via a backwards induction argument, starting with agendas of full length, and then working back to smaller agendas.

Third, the set $C E(a)$ is not always uniquely determined. That is, there may be several different sets which satisfy conditions (CE1) and (CE2); even when consistency (CE3) is imposed. This stems from the fact that the conditions are designed to be weak, to specify conditions that an equilibrium set should satisfy, but not so strong as to always uniquely determine that set. Again, this traces back to our deliberate avoidance of any reliance on an ad hoc formulation of the proposal process. To see an easy example of the potential multiplicity of equilibrium continuations, consider a somewhat degenerate voting rule as follows.

Example 2 Multiple Collections of Sets of Continuation Equilibria:

Under $V$ the outcome is always the second alternative proposed in the agenda (or the first if the agenda is a singleton), regardless of the preference profile. So $V(a)=a_{2}$ if $a \in A^{k}$ with $k \geq 2$ and $V(a)=a_{1}$ if $a \in A^{1}$.

This is a peculiar voting rule, but one that allows for a simple illustration of the multiplicity of equilibria. Note that in this case, $C E(a)=A(a)$ is uniquely determined for any $a \in A^{k}$ for $k \geq 2$. This follows since once the second alternative has been proposed the outcome is already determined and the rest of the agenda is completely irrelevant and so under (CE2) and (CE3) all continuations are then equilibria. Now consider the outcome that is proposed in the second place in the agenda. In particular,

\footnotetext{
${ }^{12}$ Of course, this is similar to a definition such as subgame perfect equilibrium where continuation strategies must be specified for each possible subgame.
} 
let $X=\{w, x, y, z\}$ and consider a preference profile where some agents have preferences $z, y, x, w$, and others have preferences $z, x, y, w$; where the ordering specifies the strict preferences where $w$ is the worst alternative. Consider starting at the agenda $a=\{w\}$. So, $w$ is the status quo. Conditions (CE1) and (CE2) have only very weak implications here: it cannot be an equilibrium to stop at $\{w\}$. Beyond that, they allow for a variety of continuation equilibrium sets. Once consistency is added, however, things are tied down to a greater degree. In particular, there are two sets which satisfy (CE1), (CE2) and (CE3). The first such set consists of all extensions of $a$ with $z$ in second place (i.e., $C E(a)=A((w, z)))$; and the second such set consists of all extensions of $a$ with any of $x, y$, or $z$ in second place (i.e., $C E(a)=A((w, x)) \cup A((w, y)) \cup A((w, z)))$.

In this example, consistency (CE3) still does not uniquely tie things down. One might argue that extensions of $(w, z)$ are really the only sensible equilibrium continuations in the above example, as they are unanimously preferred to proposals $x$ and $y$. One may wish to impose such additional conditions on the notion of equilibrium (and we discuss this more fully in Section 6). However, as we shall see, if we restrict attention to more sensible voting rules, such as those which satisfy a Pareto efficiency condition, consistency will already tie things down uniquely without the imposition of any additional conditions.

Given the potential multiplicity of collections of equilibria, we now show that in many cases of interest the set of continuation equilibria is in fact uniquely determined under consistency. This allows us to develop an equivalent definition that is not self-referential.

\section{Equilibrium Agendas for Pareto Efficient Voting Rules}

An alternative $x \in B \subset X$ is Pareto efficient relative to $B$ if there does not exist $y \in B$ such that $y R_{i} x$ for all $i \in N$ and $y P_{j} x$ for some $j \in N$.

$V$ is Pareto efficient if $V(a)$ is Pareto efficient relative the alternatives in $a$ for each $a \in A$.

Given a collection $\{C E(a)\}_{a \in A}$ and any $a \in A$, let $P E^{+}(a)$ denote the set of agendas in $C^{+}(a) \cup a$ that result in Pareto efficient alternatives (considering all of $X$ ).

Theorem 3 For any preference profile $P \in \mathcal{P}$ and Pareto efficient voting rule $V$ and collection of sets of continuation equilibria $\{C E(a)\}_{a \in A}, V\left(a^{\prime}\right)$ is Pareto efficient (considering all alternatives) for all $a$ and $a^{\prime} \in C E(a) .{ }^{13}$ Moreover, if consistency is satisfied,

\footnotetext{
${ }^{13}$ Theorem 3 also holds if one replaces Pareto efficiency everywhere by weak Pareto efficiency, where an alternative $x \in B \subset X$ is weakly Pareto efficient relative to $P$ and $B$ if there does not exist $y \in B$ such that $y P_{i} x$ for all $i \in N$. This weakens the assumptions of the theorem, but then also the conclusions.
} 
then $\{C E(a)\}_{a \in A}$ is uniquely defined and described by

$$
C E(a)= \begin{cases}P E^{+}(a) & \text { if } V(a) R_{i} V\left(a^{\prime}\right) \forall i \text { and } a^{\prime} \in C^{+}(a) \\ P E^{+}(a) \backslash a & \text { otherwise. }\end{cases}
$$

The first result in Theorem 3 is that equilibrium agendas of Pareto efficient voting rules must result in outcomes that are Pareto efficient overall. This conclusion is not quite as obvious as it seems. For instance, it could be that $x$ is Pareto dominated by $y$, but that $V\left(a^{\prime}\right) \neq y$ for all $a^{\prime} \in A(a)$. This means that since $y$ is never in the range of $V$, it does not threaten $x$. The proof uses the fact that if $y$ is added to an agenda containing $x$, then the outcome cannot be $x$ and must instead be some other outcome that some voter prefers to $x$. Building on this reasoning we rule out equilibrium agendas leading to $x$. The details are provided in the proof in the appendix.

The second result in Theorem 3 is that under consistency the continuation equilibria of Pareto efficient voting rules are uniquely determined and described by a simple algorithm.

While Theorem 3 applies to efficient voting rules, some of the reasoning that lies behind it can be used to understand outcomes of inefficient rules. The following Lemma is one that is a key to the proofs of several results, and also can be applied to inefficient voting procedures.

Lemma 4 Consider any $P \in \mathcal{P}, a \in A$, and voting rule $V$. Suppose that $\cup_{b \in A^{\text {Full }}(a)} V(b) \subset$ $D$, for some $D \subset X$ such that if $x, y \in X$, and $x \notin D, y \in D$ then $\exists i \in N$ such that $y P_{i} x$. Under (CE1) and (CE2), $V\left(a^{\prime}\right) \in D$ for any $a^{\prime} \in C E(a)$.

The idea behind the lemma is simple - if stopping at some agenda will lead to an outcome outside of $D$, then there is some agent $i$ who can gain by adding to the agenda and getting some equilibrium continuation; and hence the only potential equilibrium agendas must result in outcomes in $D$. Setting $D$ equal to the set of Pareto efficient alternatives results in the first conclusion in Theorem 3. Setting $D$ equal to other sets, such as the top-cycle, uncovered set, etc., can also lead to interesting conclusions as we shall see below.

\section{Full-Agendas Sets}

The implications of Theorem 3 are even stronger when preferences satisfy a mild restriction.

Let $\mathcal{P}^{*}$ be the set of all profiles satisfying the restriction:

$$
\forall x, y \in X, \exists i \in N \text { such that } x P_{i} y \text { or } y P_{i} x .
$$

So, $\mathcal{P}^{*}$ is the set of profiles such that it is never the case that all individuals are indifferent between some pair of alternatives $x, y$. Of course, this condition is satisfied 
when individual preferences are strict, but also holds more generally including where some transfers or distribution of resources are possible. In this case, we obtain a characterization of continuation equilibrium outcomes that does not even require an inductive definition. ${ }^{14}$

Given a voting rule $V$ and a status-quo, let the Full-Agendas Set $F A_{V}(a)$, be defined by

$$
F A_{V}(a)=\cup_{a^{\prime} \in A^{\mathrm{Full}}(a)} V\left(a^{\prime}\right) .
$$

These are all the outcomes that can be reached when voting over the full-agenda extensions of $a$.

A prominent example of a full-agendas set is the Banks Set (as detailed below), where $V$ is sophisticated voting by successive elimination. The Full-Agendas Set definition here is the analog for any voting rule.

Theorem 5 Consider a Pareto efficient voting rule $V$ and profile of preferences $P \in$ $\mathcal{P}^{*}$. If $\{C E(a)\}_{a \in A}$ is a collection of sets of continuation equilibria, then the outcomes corresponding to continuation equilibria following some agenda a are a subset of those that can be found by considering only full length agendas that are extensions of $a$. That $i s$,

$$
\cup_{a^{\prime} \in C E(a)} V\left(a^{\prime}\right) \subset F A_{V}(a) .
$$

If in addition consistency is satisfied, then these sets are equal:

$$
\cup_{a^{\prime} \in C E(a)} V\left(a^{\prime}\right)=F A_{V}(a) .
$$

Theorem 5 shows how powerful the implications of the simple stopping condition are. It states that the equilibrium outcomes correspond to those where complete agendas are considered. The idea behind this follows an inductive proof. Suppose this is true once an agenda is of length $k$ or more. Now suppose that some agenda of length $k-1$ is an equilibrium agenda and results in an outcome that differs from all full length agendas, and thus all continuation equilibria if any outcome is added. Given Pareto efficiency, some agent must prefer some outcome of a longer agenda that is a continuation equilibrium if some alternative is added to the current agenda to that of stopping. Then (CE2) implies that stopping cannot be an equilibrium.

The proof of Theorem 5 is in the Appendix. The second half of the proof actually follows from a stronger claim which does not invoke Pareto efficiency of the voting procedure. Since this is of independent interest, we state it here. ${ }^{15}$

\footnotetext{
${ }^{14}$ To see an example of why this condition is needed in the theorem, consider a situation where all voters are indifferent between all alternatives, and when there is a tie in voting the last item in the agenda wins. It can be an equilibrium to stop at any agenda (including the status quo) given full indifference, and yet the status-quo can never be reached by a full length agenda.

${ }^{15}$ In fact we prove stronger statements in the appendix, showing that even for inefficient voting rules there is a minimal consistent set of equilibria (in terms of set inclusion), which corresponds to the definition under the algorithm above. It is under Pareto efficiency that this must coincide with all consistent sets of equilibria.
} 
Claim 6 For any voting procedure $V$, preference profile $P \in \mathcal{P}$ and $a \in A$, if $\{C E(a)\}_{a \in A}$ is a collection of sets of continuation equilibria satisfying consistency, then any Pareto efficient alternative that can be reached via some full length continuation of a is an equilibrium continuation outcome following a at $P .^{16}$

\section{Applications to Specific Voting Rules and Settings}

In order to demonstrate the implications and usefulness of Theorems 3 and 5, we apply them to a number of settings including some prominent ones.

\subsection{Order Independent Voting Rules}

A voting rule $V$ is order independent if $V(a)=V\left(a^{\prime}\right)$ whenever $\{x \in a\}=\left\{x \in a^{\prime}\right\}$.

Order independent voting rules are those for which the ordering of the agenda does not matter. Neutral voting rules are order independent, but there are also important order independent voting rules that are non-neutral. Consider the following example: candidates are people who are seeded according to their age (or experience, rank, etc.). Regardless of the order in which they are proposed or nominated, the two youngest candidates are voted upon, then the winner of that vote is pitted against the next youngest, etc.. This rule is independent of the order in which the candidates are proposed, and yet it is still a sequential rule and is clearly not neutral. Therefore, we emphasize that "order independence" refers only to the order of the agenda and does not mean that the voting rule itself is not based on some implicit ordering of alternatives.

Note that for any order independent voting rule, $V(a)=V\left(a^{\prime}\right)$ for any $a$ and $a^{\prime}$ in $A^{m}$. With an abuse of notation, we write this outcome as $V(X)$.

The following is a direct corollary of Theorem 5 .

Corollary 7 For any Pareto efficient and order independent voting rule $V$, preference profile of preferences $P \in \mathcal{P}^{*}$, collection of sets of continuation equilibria $\{C E(a)\}_{a \in A}$ (i.e., satisfying (CE1) and (CE2)), and agenda $a \in A$, there is a unique continuation equilibrium outcome

$$
\cup_{a^{\prime} \in C E(a)} V\left(a^{\prime}\right)=V(X)
$$

An important remark about Corollary 7 is that it does not require consistency, but follows from (CE1) and (CE2) in the definition.

The following example shows how Borda's rule is covered under Corollary 7.

\footnotetext{
${ }^{16}$ Since we show in the appendix that there is a minimal consistent set of equilibria (in terms of set inclusion), this must hold for the minimal consistent set of equilibria.
} 
Example 8 Borda Voting.

Voters' preferences are :

- $x P_{1} w P_{1} y P_{1} z$

- $x P_{2} w P_{2} y P_{2} z$

- $z P_{3} w P_{3} y P_{3} x$

Voting is (sincere) voting according to Borda's rule. An alternative receives three points for a first place ranking in a voter's preferences, two points for a second place ranking, one point for a third place ranking, and no points for a fourth place ranking; and the alternative with the highest score is the outcome (with ties broken according to any deterministic rule). These are adjusted for the restricted ranking if some subset of alternatives is considered.

This is a Pareto efficient and order independent voting rule.

It is easily checked that $w$ wins whenever it is on the agenda. Also, $x$ wins if it is present but $w$ is not. If just $y$ and $z$ are present, then $y$ wins. $z$ only wins if it is the only proposed alternative.

Corollary 7 implies that the outcome of any equilibrium agenda must be $w$ in this example. Indeed, it is easily seen that no agenda leading to $y$ or $z$ can be an equilibrium, as adding $w$ to the agenda will lead to a continuation equilibrium outcome of $w$ which would be preferred over $y$ or $z$ by some agent. Similarly, if an agenda leads to $x$, then adding $w$ will lead to a continuation equilibrium of $w$, which is better for voter 3 than $x$.

\subsection{Tournaments and Top Cycle Consistent Rules}

The following definitions are useful in some of the remaining applications.

\section{Tournaments}

In many contexts, the preferences of the voters can be summarized (even for strategic purposes) by the majority voting relation that is induced over pairs of alternatives. A tournament is a binary relation that summarizes the important aspects of voters' preferences in some contexts. ${ }^{17}$ More formally, the majority voting tournament is defined as follows.

\footnotetext{
${ }^{17}$ See Laslier [11] for an illuminating account of the principal results in the vast literature on tournaments.
} 
Given $P \in \mathcal{P}$, denote by $T(P)$ the binary relation defined by

$$
x T(P) y \Leftrightarrow \#\left\{i \in N: x P_{i} y\right\}>\#\left\{i \in N: y P_{i} x\right\}
$$

Again, we omit the notation on $P$ and simply write $T$.

$T$ is always asymmetric and if $n$ is odd and individual preferences are strict then $T$ is complete. If we break ties in some deterministic manner, then even in cases with an even number of voters or indifferences $T$ is also complete, and therefore a tournament (an asymmetric and complete binary relation). In what follows, unless specified otherwise, we will assume that ties are broken so that $T$ is complete. $T$ is referred to as the majority tournament (induced by $P$ ).

\section{The Top Cycle}

As the majority tournament is not necessarily transitive, it can have cycles. A prominent cycle that we refer to in the sequel is the top cycle associated with a tournament.

The top cycle of $T$, denoted by $T C(X, T)$ is the set $\left\{x \in X: \forall y \in X, \exists x_{1}, \ldots, x_{k}\right.$ in $X$ such that $x_{1}=$ $x, x_{k}=y$ and $\left.x_{i} T x_{i+1} \forall i=1, \ldots, k-1\right\}$ i.e. the set of alternatives that can reach any other alternative in $X$ via a $T$-chain of arbitrary length. For subsets of alternatives, $B \subset X$, there is a corresponding definition and we denote that set $T C(B, T)$. When there is no $B \subset X$ indicated, then we are referring to the top cycle relative to $X$, and we use the notation $T C(a, T)$ to denote the top cycle relative to the set of alternatives in the agenda $a$ under the tournament $T$.

A voting rule is top cycle consistent at a tournament $T$ if $V(a) \in T C(a, T)$ for any $a \in A$.

\section{Condorcet Winners and Consistency}

An alternative $\{x\}$ is a Condorcet winner relative to $B \subset X$ if $T C(B, T)=\{x\}$. That is, a Condorcet winner is an alternative that beats every other alternative in $B$ under $T$.

A voting rule $V$ is Condorcet consistent if $V(a)$ selects a Condorcet winner whenever one exists relative to $T$ and the alternatives in $a$.

\subsection{Equilibrium Agendas for Top Cycle and Condorcet Con- sistent Voting Rules}

If the voting procedure $V$ arises from strategic voting on a binary tree, then it follows from McKelvey and Niemi (1978) that $V$ is top cycle consistent. Thus, the following proposition covers a wide variety of applications. 
Proposition 9 Consider a $P$ such that $T(P)$ is a well-defined tournament and a collection of sets of continuation equilibria $\{C E(a)\}_{a \in A}$ (i.e., satisfying (CE1) and (CE2)). If $V$ is top cycle consistent, then all equilibrium outcomes following any agenda are in the (overall) top cycle. Moreover, if $V$ is Condorcet consistent and there exists a Condorcet winner $x$ at $P$, then all of the equilibrium continuations from any agenda lead to $x$.

Again, remark that Proposition 9 does not require consistency (CE3).

The second statement does not quite follow from the first, since Condorcet consistency does not imply top cycle consistency. The proof of Proposition 9 is straightforward for the case where the preference profiles in $\mathcal{P}^{*}$ are strict and the voting rule is Pareto efficient. Then, from Theorem 5 we know that the equilibrium outcomes coincide with those that are full agendas and extensions of the starting agenda. These must select from the top cycle. The proof when the preference profiles are not necessarily in $\mathcal{P}^{*}$ or the voting rule is inefficient is slightly more complicated, as then Theorem 5 cannot be applied. The proof is still relatively short and appears in the appendix.

A direct corollary of Proposition 9 is that all equilibrium agendas in a setting with single-peaked preferences and a Condorcet consistent voting rule lead to the outcome of the median of the voters'

peaks.

\subsection{Voting by Successive Elimination and Equilibrium Agendas}

The voting procedure of voting by successive elimination is defined as follows. Consider some agenda $a \in A$ and let $a=\left(x_{1}, \ldots, x_{k}\right)$. In the successive elimination procedure, a vote is first taken to eliminate either $x_{k}$ or $x_{k-1}$. The 'winning' alternative from the first round is compared to $x_{k-2}$, and a vote is taken to eliminate either surviving alternative from the first vote or $x_{k-2}$, and so on. After $(k-1)$ comparisons, the last surviving alternative is declared to be the voting outcome.

At each stage, the elimination of one alternative is according to majority voting. This is well-specified when $T$ is complete. However, in cases where there are ties under the majority preference relation, either resulting from personal indifferences or from an even number of voters, $T$ is not complete. In this case, voting by successive elimination needs to be more completely specified.

We do so as follows. At each stage allow individuals to vote for one of the two alternatives or to abstain (in the case where they may be indifferent). In case of a tie in the voting between alternatives $x_{i}$ and $x_{j}, x_{i}$ is elected if and only if $x_{i}$ comes before $x_{j}$ in the ordering of voting $(i<j)$. This favors alternatives proposed earlier in the agenda under ties, which is a natural way to break ties (given that they have not already been broken under $T$ ). 
At the last stage of voting, if the voting boils down to a comparison of $x$ and $y$ where $x$ precedes $y$ in the successive elimination procedure, then $x$ wins if not $y T(P) x$ and $y$ wins otherwise.

However, in order to determine the eventual voting outcome, it is also necessary to describe how voters act. We first examine the case where they vote strategically at each stage, and so focus on the sophisticated voting outcome of this binary voting procedure. This is the outcome under the iterative elimination of weakly dominated strategies that has been well-studied (see Shepsle and Weingast (1984) for the algorithm identifying the outcome). ${ }^{18}$

Let $S(a)$ denote the sophisticated voting outcome under voting by successive elimination on agenda $a$, as described above.

\section{The Banks Set}

The Banks set is then the Full-Agendas Set for sophisticated voting by successive elimination (denoted $S)$ :

$$
B S(a)=F A_{S}(a) \cdot{ }^{19}
$$

\section{Equilibrium Agendas and Voting by Successive Elimination}

Given that sophisticated voting by successive elimination is a Pareto efficient voting rule, we have the following corollary of Theorem 5 .

Corollary 10 Consider a collection of sets of continuation equilibria $\{C E(a)\}_{a \in A}$ (satisfying (CE1) and (CE2)) and any profile of preferences $P \in \mathcal{P}^{*}$. For all $a \in A$,

$$
\cup_{a^{\prime} \in C E(a)} S\left(a^{\prime}\right) \subset B S(a),
$$

and if consistency (CE3) is also satisfied, then

$$
\cup_{a^{\prime} \in C E(a)} S\left(a^{\prime}, P\right)=B S(a) .
$$

Note that the result above also holds if we set the starting agenda $a$ to be the emptyset. $^{20}$

\footnotetext{
${ }^{18}$ The Shepsle-Weingast algorithm was defined for the case where $T$ is complete. Our procedure of breaking possible ties in the majority preference relation coming earlier in the ordering $a$ ensures that the sophisticated outcome can be derived from a straightforward variation on the algorithm derived by Shepsle and Weingast.

${ }^{19}$ Banks' (1985) definition was not relative to a status-quo agenda. Simply setting $a=\emptyset$ provides his original definition.

${ }^{20} \mathrm{An}$ easy way to see this is simply to extend the set of alternatives to include some $x_{0}$ such that all alternatives are preferred to $x_{0}$ by all agents under $P$, and then set $a=\left\{x_{0}\right\}$ and then apply the theorem as it stands.
} 
Corollary 10 states that not only does the Banks' set capture the set of outcomes that could arise from arbitrary full length agendas, but that these are also precisely the set of potential equilibrium outcomes when the agendas are endogenous.

While Corollary 10 provides a precise characterization of equilibrium agenda outcomes for an important voting procedure, it is still useful to show that this characterization completely ties down the outcome in some interesting cases. We now show this in the context of an interesting "pork barrel politics" setting. In particular, even though in some cases the top cycle of the majority voting relation may be very large, the Banks set, and thus the set of equilibrium agenda outcomes, can be a singleton.

\subsection{Voting over Projects}

Ferejohn, Fiorina, and McKelvey (1987) consider the following model. $N$ is a set of legislators (with $n$ odd), each of whom has a project for their constituency. The projects have value only for their constituents, but the cost of a project, if it is undertaken, is split evenly among all constituencies. ${ }^{21}$ Ferejohn, Fiorina, and McKelvey assume that projects have different costs, so as to ensure that $T(P)$ is complete, but that is not assumed here (as we can extend their result given our procedure for breaking ties).

So, this is a model of pure "pork-barrel" politics. Here the set of alternatives $X$ is simply a list of which projects are undertaken, and so $X=\{0,1\}^{n}$. Voting over an agenda is done by sophisticated voting by successive elimination.

Given this setting, legislators' preferences take a specific form. Their favorite alternative is to have their own project undertaken and no other projects undertaken. Beyond the decision concerning a legislator's own project, he or she simply prefers to minimize the costs of the other projects undertaken. The critical freedom in the preferences is in the relative costs of projects, which determines which projects a legislator might tolerate being undertaken in conjunction with his or her own, before the cost becomes so high that he or she would prefer to have none built at

all.

An interesting aspect of the Ferejohn, Fiorina, and McKelvey (1987) model is the importance of a status quo. The status quo is that no projects are undertaken. Applying our equilibrium approach to this model is of particular interest as it shows how the status quo can tie down equilibrium agendas, and illustrates why we have been careful to defined continuation equilibrium concepts that allow for a status quo. It also shows that the conclusions reached by Ferejohn, Fiorina, and McKelvey (1987) without an equilibrium analysis, are robust to an equilibrium formulation.

\footnotetext{
${ }^{21}$ This assumption is not necessary. All that matters is that the legislators agree about the relative rankings of how costly (in terms of how much they each pay) different projects are.
} 
Let $X^{*}$ denote the set of $x \in X$ that (i) undertake exactly $\frac{n+1}{2}$ projects, (ii) are as cheap as any other choice of exactly $\frac{n+1}{2}$ projects, and (iii) are such that $x T 0$. This may be empty.

Corollary 11 Consider any profile of admissible preferences $P \in \mathcal{P}$ and collection of sets of continuation equilibria $\{C E(a)\}_{a \in A}$ (satisfying (CE1) and (CE2)) in the extension of the Ferejohn, Fiorina, and McKelvey setting where some projects may have identical costs. $^{22}$

$$
\cup_{a^{\prime} \in C E\left(a_{0}\right)} S\left(a^{\prime}\right)= \begin{cases}X^{*} & \text { if } X^{*} \neq \emptyset \\ 0 & \text { otherwise. }\end{cases}
$$

Here the equilibrium agendas result in collections of projects corresponding to majorities of minimal size and which choose the cheapest projects. This minimal winning size is an interesting qualitative feature which has been extensively discussed in various areas of political science since Riker (1962). The proof of Corollary 11 appears in the appendix.

Let us emphasize here that the equilibrium predictions here are quite narrow. For many natural preference profiles, the Top-Cycle here is the entire set of alternatives, and in fact the Uncovered Set is nearly all of the alternatives. Thus, equilibrium agenda reasoning narrows the predictions dramatically from what one might predict simply by examining the tournament itself and a set based on the top-cycle, uncovered set, etc.. This also shows that in situations where a chaos-theorem type of reasoning would say that no prediction is possible, equilibrium considerations can still be quite powerful and predictive.

To see this explicitly, consider a situation where there are three different constituencies. Suppose that project 1 is cheaper than project 2 is cheaper than project 3 . To keep things simple, also suppose that any legislator prefers to have all projects built to having none built. So for instance, $[1,1,1]$ (all projects being built) is preferred to $[0,0,0]$ (no projects being built). This results in the following majority voting relationship:

$$
\begin{aligned}
& {[0,0,0] \text { beats }[1,0,0],[0,1,0] \text {, and }[0,0,1]} \\
& {[1,0,0] \text { beats }[0,1,0],[0,0,1],[1,1,0] \text { and }[1,0,1]} \\
& [0,1,0] \text { beats } 0,0,1],[1,1,0] \text { and }[0,1,1] \\
& {[0,0,1] \text { beats }[1,0,1] \text { and }[0,1,1]} \\
& {[1,1,0] \text { beats }[0,0,0],[1,1,1],[0,1,1],[1,0,1], \text { and }[0,0,1]} \\
& {[1,0,1] \text { beats }[0,0,0],[1,1,1],[0,1,1], \text { and }[0,1,0]} \\
& {[0,1,1] \text { beats }[0,0,0],[1,1,1] \text { and }[1,0,0]}
\end{aligned}
$$

\footnotetext{
${ }^{22}$ Note that the preference profile will naturally end up in $\mathcal{P}^{*}$ under their assumptions.
} 
$[1,1,1]$ beats $[0,0,0],[1,0,0],[0,1,0]$, and $[0,0,1]$.

This is pictured in the following figure

[Insert Figure 1 here]

The corresponding Top Cycle is all of the alternatives. The Uncovered set here is all of the alternatives except $[0,0,1]$. Yet the outcome identified under equilibrium agenda formation (Corollary 11) is simply the singleton $[1,1,0]$.

\subsection{Sincere Voting and an Absence of Chaos}

The previous results show that equilibrium conditions on agendas can make narrow predictions. The examples we worked out in detail so far used sophisticated voting. As much of the literature on chaos theorems (e.g., McKelvey (1979)) was restricted to sincere voting we show that equilibrium reasoning can also be predictive there. In particular, we show that even in situations where the top cycle is large (even the whole set of alternatives), considering only equilibrium agendas still narrows the set of predictions in well-defined ways.

The marriage of equilibrium agenda formation with sincere voting is a bit schizophrenic. On the one hand, we are analyzing sophisticated (forward looking) agenda formation, while on the other hand, we assume myopic voting. The reason we undertake this exercise of applying our equilibrium agenda formation to sincere voting is to revisit the chaos-theorem setting as closely as possible to understand where the departure in conclusions comes from, show the versatility of the equilibrium reasoning, and show the robustness of the claim that equilibrium reasoning in agenda formation offers narrow predictions.

While the setting we consider in this section is a finite one (see the next section for the infinite case), we can still see the essence of chaos theorems in the following way. Consider sincere voting by successive elimination, where when asked to compare any two alternatives, voters vote for the one that they prefer, not anticipating the outcome of the votes yet to come in the sequence. ${ }^{23}$ The critical observation is that for any $x \in T C(T)$

\footnotetext{
${ }^{23}$ One might also term this myopic voting. Note, however, that this corresponds to sophisticated voting under the following alternative voting rule. The closely related voting procedure for which this is sophisticated is as follows. On an agenda $a=\left(x_{1}, \ldots, x_{K}\right)$, select $x_{1}$ unless a majority votes to move on to $x_{2}$; then select $x_{2}$ unless a majority votes to move on to $x_{3}$, and so forth. Sophisticated voting on this rule can be solved as follows. If one gets to the last decision of whether or not to select $x_{K-1}$ or move on, then the vote will be a sincere vote between $x_{K}$ and $x_{K-1}$. Anticipating this, the previous vote is a sincere vote between $x_{K-2}$ and the sincere winner between $x_{K}$ and $x_{K-1}$. Rolling this back up the voting tree, this is solved exactly as a sincere vote by successive elimination.
} 
and any $k$, there exists an agenda $a \in A^{k}$, such that $V(a)=x$, where $V$ is sincere voting by successive elimination. In particular, setting $k=m$, any $x$ in the top cycle can be reached by at least one full length agenda (in fact at least two). ${ }^{24}$ This means that if we are not able to do any selection over agendas, then any alternative in the top cycle can be an outcome. ${ }^{25}$

The following example, however, illustrates that our definition of equilibrium selects from the agendas. Here only a subset of the top cycle alternatives are equilibrium outcomes, even though all alternatives (other than a unanimously bad status quo) are in the top cycle. Thus, the notion of equilibrium does preclude alternatives and makes selections from the top cycle. ${ }^{26}$

Example 12

\section{Voters' preferences are :}

- $x_{5} P_{1} x_{2} P_{1} x_{3} P_{1} x_{4} P_{1} x_{1} P_{1} x_{0}$

- $x_{4} P_{2} x_{5} P_{2} x_{1} P_{2} x_{2} P_{2} x_{3} P_{2} x_{0}$

- $x_{3} P_{3} x_{4} P_{3} x_{5} P_{3} x_{1} P_{3} x_{2} P_{3} x_{0}$

The induced tournament $T$ is that

- $x_{5}$ beats $x_{0}, x_{1}, x_{2}$, and $x_{3}$,

- $x_{4}$ beats $x_{0}, x_{1}, x_{2}$, and $x_{5}$,

- $x_{3}$ beats $x_{0}, x_{1}$, and $x_{4}$,

- $x_{2}$ beats $x_{0}$ and $x_{3}$,

- $x_{1}$ beats $x_{0}$ and $x_{2}$.

Note that here $B S\left(\left\{x_{0}\right\}\right)=\left\{x_{3}, x_{4}, x_{5}\right\}$ and $T C(X)=\left\{x_{1}, x_{2}, x_{3}, x_{4}, x_{5}\right\}$; and also that both $x_{4}$ and $x_{5}$ Pareto dominate $x_{1}$.

\footnotetext{
${ }^{24} \mathrm{~A}$ recipe is as follows. Find an ordering of the $K$ alternatives in the top cycle $x=x_{1}, x_{2}, \ldots, x_{K}$, such that $x_{i} T(P) x_{i+1}$ for each $i<K$. Such an ordering always exists. Consider any agenda where the top cycle alternatives maintain this relative ordering and the other alternatives fall in any place. Sincere voting by successive elimination will lead to $x$. The second variation is to switch the position of $x_{K}$ and $x_{K-1}$, which does not affect the outcome.

${ }^{25}$ Note that sophisticated behavior in voting by successive elimination can preclude some alternatives from the top cycle as ever being equilibrium outcomes as we already saw in Corollary 10.

${ }^{26}$ In light of Footnote 23 and Proposition 9, equilibria under sincere voting by successive elimination will always end up in the top cycle, and so the example shows it can end up being a strict subset that is selected.
} 
Under sincere voting by successive elimination, the agendas (with a status quo of $x_{0}$ ) that can lead to an outcome of $x_{1}$ are those that follow the ordering of the index of the alternatives without gaps, starting at $x_{0}$, except possibly that the last two alternatives may be switched. ${ }^{27}$

None of these are equilibrium agendas when the status quo is $a=\left\{x_{0}\right\}$ (i.e., none of these are in $C E\left(\left\{x_{0}\right\}\right)$ ). Thus, $x_{1}$ is not an equilibrium agenda outcome when $V$ is sincere voting by successive elimination.

First, it is easily checked that $\left\{x_{0}, x_{1}\right\}$ and $\left\{x_{0}, x_{2}, x_{1}\right\}$, are not continuation equilibrium agendas (i.e., stopping once they are reached), as adding $x_{5}$ will lead to an outcome of either $x_{4}$ or $x_{5}$ which are unanimously preferred to $x_{1}$; and so (CE2) is violated. Thus they could not be equilibrium agendas beginning at $x_{0}$. We can also check that the agenda $\left\{x_{0}, x_{1}, x_{3}, x_{2}\right\}$ is not a continuation equilibrium. If either $x_{5}$ or $x_{4}$ is added one obtains either $x_{3}$ as the only equilibrium outcome. ${ }^{28}$ Then it cannot be an equilibrium to stop, as voters 1 or 3 would gain by proposing either $x_{4}$ or $x_{5}$.

The agendas that remain to be checked that might lead to $x_{1}$ are those in $A\left(\left\{x_{0}, x_{1}, x_{2}\right\}\right)$. Note that for any $a^{\prime} \in A\left(\left\{x_{0}, x_{1}, x_{2}, x_{3}\right\}\right)$, the outcome is $x_{1}$, while for any $a \in A\left(\left\{x_{0}, x_{1}, x_{2}, x_{5}\right\}\right)$ the outcome $x_{4}$ or $x_{5}$. Thus, consistency (CE3) implies that if $x_{1}$ is an equilibrium outcome following $\left\{x_{0}, x_{1}, x_{2}\right\}$, then also $x_{4}$ or $x_{5}$ is an equilibrium outcome following $\left\{x_{0}, x_{1}, x_{2}\right\}$, and that $x_{1}$ can only come from proposing $x_{3}$ next. Also, note that $x_{3}$ is not an outcome under any agenda in $A\left(\left\{x_{0}, x_{1}, x_{2}\right\}\right)$ as it loses to $x_{2}$, and also $x_{2}$ and $x_{0}$ are never outcomes under any agendas in $A\left(\left\{x_{0}, x_{1}, x_{2}\right\}\right)$. Then by (CE3) it follows that $x_{1}$ is not an equilibrium outcome following $\left\{x_{0}, x_{1}, x_{2}\right\}$, and those equilibrium outcomes are a subset of $\left\{x_{4}, x_{5}\right\}$.

The example uses the fact that agendas that lead to $x_{1}$ must have $x_{1}$ in one of the first three places in the agenda. This always leaves additional alternatives that can be proposed that would lead to other outcomes, and the preference for some of these other outcomes prevents the specific agendas leading to $x_{1}$ from being equilibrium agendas.

Thus, chaos is avoided and we have predictions that we end up inside a strict subset of the top cycle.

In fact, we also have a "lower bound" on the set of possible outcomes of sincere voting under sequential elimination - Claim 1 in the appendix implies that all Pareto optimal elements in the top cycle can be supported as outcomes of continuation equilibria.

Finally, we show that equilibrium agendas under sincere voting under sequential elimination can lead to Pareto inefficient outcomes.

\footnotetext{
${ }^{27}$ Explicitly, the agendas leading to an outcome of $x_{1}$ are $\left\{x_{0}, x_{1}, x_{2}, x_{3}, x_{5}, x_{4}\right\},\left\{x_{0}, x_{1}, x_{2}, x_{3}, x_{4}, x_{5}\right\}$, $\left\{x_{0}, x_{1}, x_{2}, x_{3}, x_{4}\right\},\left\{x_{0}, x_{1}, x_{2}, x_{4}, x_{3}\right\},\left\{x_{0}, x_{1}, x_{2}, x_{3}\right\},\left\{x_{0}, x_{1}, x_{3}, x_{2}\right\},\left\{x_{0}, x_{1}, x_{2}\right\},\left\{x_{0}, x_{2}, x_{1}\right\}$, and $\left\{x_{0}, x_{1}\right\}$.

${ }^{28} \mathrm{By}$ reasoning similar to that above, it is easily checked that if $x_{5}$ is added next, then $x_{4}$ would be also added next in equilibrium.
} 


\section{Example 13}

Let $X=\left\{x_{0}, x_{1}, x_{2}, x_{3}, x_{4}, x_{5}\right\}$. The status quo is $x_{0}$. There are 3 individuals, with preferences given below.

- $x_{1} P_{1} x_{2} P_{1} x_{5} P_{1} x_{3} P_{1} x_{4} P_{1} x_{0}$

- $x_{5} P_{2} x_{3} P_{2} x_{4} P_{2} x_{1} P_{2} x_{2} P_{1} x_{0}$

- $x_{4} P_{3} x_{1} P_{3} x_{5} P_{3} x_{2} P_{3} x_{3} P_{1} x_{0}$

The induced tournament $T$ is :

- $x_{1}$ beats $x_{0}, x_{2}, x_{3}$, and $x_{5}$,

- $x_{2}$ beats $x_{0}$ and $x_{3}$,

- $x_{3}$ beats $x_{0}$ and $x_{4}$,

- $x_{4}$ beats $x_{0}, x_{1}$ and $x_{2}$,

- $x_{5}$ beats $x_{0}, x_{2}, x_{3}$ and $x_{4}$.

Note that $x_{2}$ is Pareto dominated by $x_{1}$.

Let us argue that $a=\left(x_{0}, x_{2}, x_{3}, x_{4}, x_{1}\right)$ which results in $x_{2}$ is in $C E\left(\left\{x_{0}\right\}\right)$. Since adding $x_{5}$ makes no difference to the outcome, this is an equilibrium agenda once $a$ is reached. Moving back, $a$ is an equilibrium continuation of $\left(x_{0}, x_{2}, x_{3}, x_{4}\right)$. If instead $x_{5}$ is added so to get $\left(x_{0}, x_{2}, x_{3}, x_{4}, x_{5}\right)$, then it will not be an equilibrium to stop as the outcome would be $x_{5}$ and agent 1 would prefer to add $x_{1}$ so that the outcome would again be $x_{2}$. Thus, all equilibrium continuations of $\left(x_{0}, x_{2}, x_{3}, x_{4}\right)$ lead to $x_{2}$.

Next, note that $a^{\prime}=\left(x_{0}, x_{2}, x_{3}, x_{5}, x_{4}, x_{1}\right)$ results in $x_{5}$, which is voter 2 's favorite. Thus, we know that it is possible to reach $\left(x_{0}, x_{2}, x_{3}\right)$. Then under (CE3), voter 1 is willing to propose $x_{4}$ expecting the continuation of $a$ leading to $x_{2}$, given that there is another continuation equilibrium leading to $x_{5}$. As argued above, we then have $a$ as an equilibrium continuation once $\left(x_{0}, x_{2}, x_{3}, x_{4}\right)$ has been reached.

Thus $x_{2}$ is an equilibrium outcome when the status quo is $x_{0}$. 


\subsection{Handling Infinities}

Our discussion so far has focused on a finite set of alternatives $X$. We now demonstrate how our analysis works in more general settings where the set of alternatives may be infinite. An important first remark is that the definitions we have for continuation equilibria, (CE1)-(CE3), can be applied directly to the infinite case without modification.

However, there are new challenges that arise in applying the definition of equilibria in infinite settings, which we will address below. One challenge is whether or not to define voting rules on infinite sequences of alternatives, and if it is done, how to do it. There are different ways that this might be done and the specific choice of how to do it is usually specific to the setting in question. Another challenge is to establish existence of equilibrium sets. In the finite case existence was straightforward as we could follow a simple backward induction argument. In the infinite case the issue is more subtle and will require using some characteristics of the setting being analyzed. A third challenge is that even when collections of sets of agenda equilibria can be shown to exist, it may still be hard to get a handle on a characterization of them as, again, a simply backward induction approach is precluded.

Nevertheless, despite these challenges the definitions turn out to be quite manageable in several ways as we now show.

Consider an infinite $X$. Let $A=\cup_{k} A^{k}$ be now the set of arbitrary length finite agendas. $^{29}$

Given a voting rule $V$, say that an agenda $a \in A$ is maximal if $V\left(a^{\prime}\right)=V(a)$ for all $a^{\prime} \in A(a)$. Denote the set of maximal agendas for $V$ that are the continuation of some $a$ by $M_{V}(a)$.

The analogue of Theorem 5 now follows.

First, we show that when the set of maximal agendas is nonempty, then there exists a natural set of continuation equilibria.

Lemma 14 Consider an infinite $X$, a profile of preferences $P \in \mathcal{P}^{*}$, and a Pareto efficient voting rule $V$ such that $M_{V}(a)$ is nonempty for all agendas $a \in A$. Then there exists a collection of sets of continuation equilibria $\{C E(a)\}_{a \in A}$ satisfying (CE1)-(CE3), which is to set $C E(a)=M_{V}(a)$ for each $a$.

Lemma 14 leaves open the question of when $M_{V}(a)$ is nonempty for all agendas. This is easy to check in some cases as when there is a Condorcet winner, and can also be

\footnotetext{
${ }^{29}$ Here we could extend a voting rule $V$ to be defined over infinite agendas, but it is not necessary. For the interested reader, one way of defining $V$ over infinite agendas is as follows. Consider an infinite $a$, and let $a_{k}$ be the agenda consisting of the first $k$ proposed alternatives. If there exists some $K$ such that $V\left(a_{k}\right)=V\left(a_{K}\right)$ for all $k \geq K$, then define $V(a)=V\left(a_{K}\right)$. Have some rule for assigning $V(a)$ otherwise, such as fixing a status quo $x$ and if voting never resolves itself then the status quo stays in place.
} 
verified in some settings such as the three person divide-the-dollar game analyzed by Penn (2001). We leave the exploration of more subtle conditions guaranteeing nonemptiness for future research.

Now we can establish the analog of Theorem 5 for the infinite case.

Theorem 15 Consider an infinite $X$, a profile of preferences $P \in \mathcal{P}^{*}$, and a Pareto efficient voting rule $V$ such that $M_{V}(a)$ is nonempty for all agendas a $\in A$. For any collection of sets of continuation equilibria $\{C E(a)\}_{a \in A}$ (satisfying (CE1) and (CE2)), and any finite $a \in A$,

$$
\cup_{a^{\prime} \in C E(a)} V\left(a^{\prime}\right) \subset \cup_{b \in M_{V}(a)} V(b),
$$

and if consistency (CE3) is also satisfied, then

$$
\cup_{a^{\prime} \in C E(a)} V\left(a^{\prime}\right)=\cup_{b \in M_{V}(a)} V(b) .
$$

The proof of Theorem 15 is provided in the appendix. Here, we provide the basic intuition. The proof of Theorem 5 exploited the possibility of backward induction from agendas $a \in A^{m}$. Notice that if $a$ is a maximal agenda, then all $b \in A(a)$ can essentially be ignored. Hence, maximal agendas play the same role in the infinite setting that agendas in $A^{\text {Full }}(a)$ play in the finite environment.

\section{Discussion of the Definition of Equilibrium}

\section{Proposals to Stop the Agenda or Seconds to Continue an Agenda}

Some procedures may allow an individual to propose a motion that voting take place immediately on the existing agenda. This motion is voted "yes" or "no", and a majority support can stop the existing agenda. Alternatively, a procedure may require at least two agents to support a proposal in order to add it to the agenda.

If either of these variations are present, it makes no difference to the analysis, at least under sophisticated voting by successive elimination. Let us offer a heuristic argument for why Corollary 16 extends in this way.

We argue by induction. It is clearly true starting at some full length agenda. Suppose it is true starting at agendas of length at least $k+1$. Consider an existing agenda $a \in A^{k}$, $S(a)=x$, and individual $i$ proposes the motion that voting take place immediately. If $i$ 's motion is defeated, then her proposal is irrelevant. On the other hand, if $i$ 's motion is accepted, then $x$ becomes the final outcome. This implies that a majority prefers $x$ to any outcome that can be obtained by some further continuation equilibrium, which from the induction step and the corollary corresponds to the outcome of some $a^{\prime} \in A(a) \cap A^{m}$. If $x$ already corresponds to such an outcome, then the claim is true. If not, then by the 
Shepsle-Weingast algorithm, there must be some alternative $y \notin a$ such that $y$ is preferred by a majority to $x$ and such that $y$ is the outcome under a continuation equilibrium $a^{\prime} \in A(a) \cap A^{m}$. This, however, implies that a majority would vote to continue rather than stop at $x$, which would be a contradiction. Thus the claim is true.

The argument for having a second agent move a proposal to make it part of an agenda is analogous, noting that if a majority prefer $y$ to $x$, then at least two agents must prefer to follow the continuation equilibrium leading to $y$ rather than stopping at $x$.

\section{Modifications of Consistency}

The notion of consistency (CE3) is one that produces a large set of equilibria relative to those which might be considered (witness Theorem 5 and Claim 6). We now consider a more stringent form of rationalizability, that in turn corresponds to a different form of consistency that includes fewer continuation equilibria.

We say that an agenda $a^{\prime}=(a, x, \ldots)$ is strongly rationalizable (relative to $a$ ) if $a^{\prime} \in C^{+}(a)$ and

there exists $i \in N$ such that for any $y \notin a$ and $y \neq x$ there exists some $a^{\prime \prime} \in C E((a, y))$ such that $V\left(a^{\prime}\right) R_{i} V\left(a^{\prime \prime}\right)$, and if $a \in C E(a)$ then also $V\left(a^{\prime}\right) R_{i} V(a)$.

Strong rationalizability only allows for an agenda $(a, x, \ldots)$ which is a continuation of $a$ to be supported only if there is some agent who does not prefer all equilibrium continuations of $(a, y)$ to those of $(a, x)$. The idea being that an agent who prefers all continuations of $(a, y)$ to those of $(a, x)$ would not propose $x$, but would instead propose $y$ (or possibly some other alternative). This differs from rationalizability, in that rationalizability allows some $i$ to propose $x$ if there is some alternative continuation that the agent finds worse; but this does not consider the fact that the agent might prefer to propose something else in $y$ 's place.

We say that a collection of sets of continuation equilibria is strongly consistent if it satisfies $^{30}$

(CE4) (Strong Consistency) If $a^{\prime} \in C^{+}(a)$ is strongly rationalizable, then $a^{\prime} \in C E(a)$. Conversely, if $a^{\prime}=(a, x, \ldots) \in C E(a)$ and either $a \in C E(a)$ or $a^{\prime \prime}=(a, y, \ldots) \in$ $C E(a)$ for some $y \neq x$, then $a^{\prime}$ is strongly rationalizable.

Let us make a couple of remarks. First, sets of continuation equilibria satisfying (CE1), (CE2), and (CE4) always exist. This can be seen by noting that starting from

\footnotetext{
${ }^{30}$ When we modify (CE3) to (CE4), we might also consider adding another condition, which was implied under (CE1), (CE2) and (CE3), but not under (CE1), (CE2) and (CE4). The condition is (5) If $(a, x, \ldots) \in C E(a)$ then $C E((a, x)) \subset C E(a)$. This is irrelevant in the discussion below.
} 
some agenda either there exists some strongly rationalizable alternative (possibly several) or else $a$ must be the equilibrium continuation. Second, from Theorem 3 we know that for Pareto efficient rules continuation equilibria satisfying strong consistency (CE4) always are a subset of those satisfying consistency (CE3). Example 1 is easily seen to be one where this is a strict subset. However, that is an inefficient voting rule. The following example shows that the selection may be strict even for sophisticated voting by successive elimination, where strong consistency results in a strict subset of the Banks' set.

Example 16

Let $X=\left\{x_{1}, x_{2}, x_{3}, x_{4}, x_{5}\right\}$ and $N=\{1,2,3\}$.

The preference profile is:

- $x_{1} P_{1} x_{3} P_{1} x_{2} P_{1} x_{4} P_{1} x_{5}$

- $x_{5} P_{2} x_{3} P_{2} x_{4} P_{2} x_{1} P_{2} x_{2}$

- $x_{2} P_{3} x_{4} P_{3} x_{5} P_{3} x_{1} P_{3} x_{3}$

Then, the induced tournament $T$ is

- $x_{4}$ beats $x_{1}$ and $x_{5}$.

- $x_{1}$ beats $x_{2}$ and $x_{3}$.

- $x_{2}$ beats $x_{4}$ and $x_{5}$.

- $x_{3}$ beats $x_{2}$ and $x_{4}$.

- $x_{5}$ beats $x_{1}$ and $x_{3}$.

Then, $B S\left(\left\{x_{0}\right\}\right)=X$. We want to show that if $C E\left(\left\{x_{0}\right\}\right)$ satisfy (CE1),(CE2) and strong consistency, then the equilibrium outcomes are $\left\{x_{1}, x_{2}, x_{5}\right\}$.

First, note that if $a \in C^{+}\left(\left\{x_{0}, x_{1}\right\}\right)$, then $S(a)=x_{4}$. For if $a \in A\left(\left\{x_{1}\right\}\right)$, the possible outcomes are in $\left\{x_{5}, x_{4}\right\}$. But since $x_{4}$ beats $x_{5}$, (CE2) implies that $S(a)=x_{4}$ if $a \in C^{+}\left(\left\{x_{0}, x_{1}\right\}\right)$.

Analogously, the following are true.

- If $a \in C^{+}\left(\left\{x_{0}, x_{2}\right\}\right)$, then $S(a)=x_{1}$.

- If $a \in C^{+}\left(\left\{x_{0}, x_{5}\right\}\right)$, then $S(a)=x_{2}$.

- If $a \in C^{+}\left(\left\{x_{0}, x_{3}\right\}\right)$, then $S(a)=x_{5}$.

- If $a \in C^{+}\left(\left\{x_{0}, x_{4}\right\}, P\right)$, then $S(a)=x_{3}$. 
The proof is completed by showing that no one wants to propose $x_{1}$ or $x_{4}$ initially.

This must be true since 1 prefers to propose $x_{2}$ initially. This guarantees choice of $x_{1}$, which is 1 's most preferred element in $X$. Similarly, 2 and 3 prefer initial proposals of $x_{3}$ and $x_{5}$ respectively.

\subsection{Efficiency under an Inefficient Voting Rule!}

It is interesting to note that equilibrium agenda formation can actually improve outcomes of some voting procedures. Here is one such example.

Example 17 Voting to Admit Members to a Society.

Consider the election of individuals to a society, as studied by Barberá, Sonnenschein, and Zhou (1991). Voters are current members of the society and they can nominate any individuals for consideration. The voting rule is then an approval rule with a quota. That is, voters can cast votes for any of the nominated individuals, and as many as they like, and then the admitted individuals are those receiving at least the required quota of votes.

To better understand this, let us consider a situation with two voters, $\{1,2\}$, and two candidates who might be considered for the society, $\left\{c_{1}, c_{2}\right\}$. In this case, the set of possible outcomes is the set of possible new admissions to the society, $X=$ $\left\{[\emptyset],\left[c_{1}\right],\left[c_{2}\right],\left[c_{1}, c_{2}\right]\right\}$. So here, [.] is an outcome that identifies $\cdot$ as the newly admitted candidates to the society. The status quo agenda is $a=([\emptyset])$.

Interestingly, such voting procedures are strategy-proof (under some restrictions on the separability of preferences - candidates are goods or bads, independent of who else might be elected); but they are not efficient.

To see why consider the following preferences of the voters

$$
\left\{c_{1}\right\} P_{1} \emptyset P_{1}\left\{c_{1}, c_{2}\right\} P_{1}\left\{c_{2}\right\}
$$

and

$$
\left\{c_{2}\right\} P_{2} \emptyset P_{2}\left\{c_{1}, c_{2}\right\} P_{2}\left\{c_{1}\right\} .
$$

So, voter 1 likes $c_{1}$ but dislikes $c_{2}$, and dislikes $c_{2}$ more than he likes $c_{1}$; that is, enough so that voter 1 would prefer that nobody be elected to having both $c_{1}$ and $c_{2}$ be elected. Voter 2's preferences are similar, except in liking $c_{2}$ and disliking $c_{1}$.

Consider the voting rule where there is a quota of 1 - so basically, if a voter likes a candidate then he can unilaterally make sure that the candidate is elected. If both 
candidates are on the agenda, then it will be a dominant strategy for each voter to vote for his preferred candidate. That is, voter 1 will vote for $c_{1}$ and voter 2 will vote for $c_{2}$. The outcome of this is $\left[c_{1}, c_{2}\right]$, so that both candidates are admitted. Yet, both voters would prefer $\emptyset$. Thus, the voting rule is inefficient.

Let us now consider endogenizing the agenda process. Equilibrium reasoning can help restore efficiency. Before providing the formal details, let us discuss the basic intuition. We begin with the status quo of no candidates being nominated. Suppose a voter nominates his preferred candidate, for instance voter 1 nominating $c_{1}$. Both voters then realize that regardless of what other nominations are made, under the dominant strategy of the voting round $c_{1}$ will be elected as voter 1 will cast an approval vote for $c_{1}$. This means that voter 2 should then also nominate $c_{2}$, since the eventual outcome will then be that both candidates are elected and voter 2 prefers this to just having $c_{1}$ elected. Now, reasoning backwards, both voters can anticipate that if one candidate is nominated, then the other one will also end up being nominated with an eventual outcome of both candidates being elected. This means that both voters should be content to stay at the status quo of having no nominations. Thus, the status quo is an equilibrium outcome and it leads to the Pareto efficient outcome of no candidates being admitted.

Looking at the equilibrium reasoning also provides for an interesting comparison between consistency and strong-consistency.

The following are $C E([\emptyset])$ 's that satisfy $(\mathrm{CE} 1)$ and $(\mathrm{CE} 2):\{([\emptyset])\}$, and $\left\{([\emptyset]),\left([\emptyset],\left[c_{1}\right],\left[c_{1}, c_{2}\right]\right)\right.$, $\left.\left([\emptyset],\left[c_{2}\right],\left[c_{1}, c_{2}\right]\right)\right\}$. The agenda $\left(\emptyset,\left[c_{1}\right],\left[c_{1}, c_{2}\right]\right)$ represents that $c_{1}$ is first nominated and then $c_{2}$ is then nominated so that the eventual vote is over both candidates $c_{1}$ and $c_{2}$. The agenda $([\emptyset])$ represents that no nominations are made.

Note that consistency identifies $C E([\emptyset])=\left\{([\emptyset]),\left(\emptyset,\left[c_{1}\right],\left[c_{1}, c_{2}\right]\right),\left(\emptyset,\left[c_{2}\right],\left[c_{1}, c_{2}\right]\right)\right\}$ as the set of possible continuation equilibria; while strong consistency results in $C E([\emptyset])=$ $\{([\emptyset])\}$. So, consistency allows for both the efficient an inefficient outcome as equilibria; while strong consistency singles out the efficient outcome as the only possible equilibrium outcome. This makes clear the difference in reasoning behind consistency and strong consistency. Consistency allows one to support the outcome of $\left[c_{1}, c_{2}\right]$ under the following reasoning: voter 1 nominates $c_{1}$ (which will eventually lead to both candidates being nominated) under the expectation that if he does not then voter 2 will nominate $c_{2}$ (which will eventually lead to both candidates being nominated). Voter 1 and voter 2 both rationalize their actions via expectations concerning what the other might do if they do not act. In contrast, strong consistency requires that one rationalize one's actions relative to the worst possible outcome under each possible action, including not acting if that is a possible equilibrium continuation. Since not acting is a possible continuation equilibrium (as implied by (CE2)), no further nomination is strongly rationalizable.

While this example might suggest that strong consistency is a "better" concept than consistency, we remark that finding the "right" refinement of equilibria in this problem may echo the problem of finding the "right" refinement in game theory - there may be no single obvious "right" answer, especially as one varies across contexts. 


\section{References}

- Austen-Smith, D. (1987), "Sophisticated Sincerity: Voting over Endogenous Agendas", American Political Science Review, 81, pp 1323-29.

- Austen-Smith, D. and J.S. Banks, (1999), Positive Political Theory I : Collective Preference, University of Michigan Press, Ann Arbor.

- Banks, J.S. (1985), "Sophisticated Voting Outcomes and Agenda Control," Social Choice and Welfare, 1, pp 295-306.

- Banks, J.S. and F. Gasmi (1987), " Endogenous Agenda Formation in Three-Person Committees", Social Choice and Welfare, 4, pp 133-152.

Barberà, S., H. Sonnenschein, and L. Zhou, (1991), "Voting by Committees," Econometrica, 59, pp 595-610.

- Baron, D. and J. Ferejohn (1989) "Bargaining in Legislatures," American Political Science Review, vol. 83, pp 1181-1206.

- Besley, T. and S. Coate (1997), “An Economic Model of Representative Democracy, Quarterly Journal of Economics, 112, pp 85-114.

- Chwe, M. S-Y. (1994), "Farsighted Coalitional stability", Journal of Economic Theory, 63, pp 299-325.

- Denzau, A., W. Riker, and K.A. Shepsle (1985), "Farquharson and Fenno: Sophisticated Voting and Home Style," American Political Science Review, vol. 79, pp 1117-1135.

- Duggan, J., (2002), "Endogenous Amendment Agendas", mimeo., University of Rochester.

- Dutta, B., M.O. Jackson and M. Le Breton (2001), "Strategic Candidacy and Voting Procedures," Econometrica, Vol. 69, pp 1013-1037.

- Dutta, B., M.O. Jackson and M. Le Breton (2002), "Voting by Successive Elimination and Strategic Candidacy," Journal of Economic Theory, Vol. 103, pp 190-218.

- Ehlers, L. and J. Weymark (2001), "Candidate Stability and Non-Binary Choice," mimeo: Université de Montréal and Vanderbilt University.

- Eraslan, H. and A. McLennan (2000), "Strategic Candidacy for Multivalued Voting Procedures" mimeo - University of Pennsylvania and University of Minnesota.

- Farquharson, R. (1969), Theory of Voting, New Haven: Yale University Press.

- Ferejohn, J., Fiorina, M. and R.D. McKelvey (1987), "Sophisticated Voting and Agenda Independence in the Distributive Politics Setting", American Journal of Political Science, 31, pp 169-194. 
- Greenberg, J. (1990), Theory of Social Situations, Cambridge University Press.

- Groseclose, T. and Krehbiel, K. (1993), "On the Pervasiveness of Sophisticated Sincerity", Chapter 10 in: W.A. Barnett, M.J. Hinich, and N.J. Schofield (eds), Political Economy: Institutions, Competition, and Representation, Cambridge University Press, pp 247-277.

- Laslier, J.F. (1997), Tournament Solutions and Majority Voting, Berlin/ Heidelberg: Springer-Verlag.

- McKelvey, R.D. (1976), "Intransitivities in Multidimensional Voting Models and some Implications for Agenda Control," Journal of Economic Theory, Vol. 12, pp 472-482.

- McKelvey, R.D. (1979), "General Conditions for Global Intransitivities in Formal Voting Models," Econometrica, Vol. 47, pp 1085-1112.

- McKelvey, R.D. and Niemi, R.G. (1978), "A Multistage Game Representation of Sophisticated Voting for Binary Procedures", Journal of Economic Theory, 18, 122 .

- Miller, N.R., Grofman, B and S.L. Feld (1990), "Cycle Avoiding Trajectories, Strategic Agendas, and the Duality of Memory and Foresight: An Informal Exposition", Public Choice, 64, pp 265-277.

- Ordeshook, P. (1993), "The Development of Contemporary Political Theory", Chapter 4 in: W.A. Barnett, M.J. Hinich, and N.J. Schofield (eds), Political Economy: Institutions, Competition, and Representation, Cambridge University Press, pp 71-104.

- Ordeshook, P. and Schwartz (1987), "Agendas and the Control of Political Outcomes", American Political Science Review, 81, pp 179-200.

- Osborne, M.J. and A. Slivinski (1996), "A Model of Political Competition with Citizen Candidates," Quarterly Journal of Economics, 111, pp 65-96.

- Penn, E.M. (2001), "A Distributive N-Amendment Game with Endogenous Agenda Formation," mimeo: Caltech.

- Plott, C. (1967), "A Notion of Equilibrium and its Possibility under Majority Rule, American Economic Review, Vol. 57, pp 787-806.

- Riker, W.H. (1962), The Theory of Political Coalitions, New Haven, Yale University Press.

- Rodriguez-Alvarez, C. (2000), "Candidate Stability and Voting Procedures," mimeo: Universitat Autonoma de Barcelona.

- Shepsle, K. and B. Weingast (1984), "Uncovered Sets and Sophisticated Voting Outcomes with Implications for Agenda Institutions," American Journal of Political Science, 28, pp 49-74. 


\section{Appendix}

Take some voting rule $V$ as given.

Let

$$
E O(a)=\cup_{a^{\prime} \in C E(a)} V\left(a^{\prime}\right)
$$

Proof of Lemma 4: We prove this by induction on the cardinality of $a$. If $a \in A^{m}$, then $C E(a)=\{a\}$, and so the assertion must be true. Suppose that for some $K<m$, the claim is true for each $k>K$ and $a \in A^{k}$. We show that the claim is true for $a \in A^{K}$.

From the induction hypothesis it follows that $V(b) \in D$ for all $b \in C^{+}(a)$, and so from (CE1) we only need to show that if $V(a) \notin D$, then $a \notin C E(a)$. Consider any $x \notin a$, and $b \in C E((a, x))$. By the induction $V(b) \in D$. Since $V(a) \notin D$, it follows from the properties of $D$ that $V(b) P_{i} V(a)$ for some $i$. (CE2) then implies that $a \notin C E(a)$, as required.

Proof of Theorem 3: Fix a Pareto efficient $V$ and a profile $P$.

The proof that $V\left(a^{\prime}\right)$ is Pareto efficient for any $a^{\prime}$ in $C E(a)$ and $a \in A$ follows directly from Lemma 4 , by letting $D$ in the lemma be the set of Pareto efficient alternatives in $X$.

To complete the proof of the theorem, we show that (CE1), (CE2) and (CE3) can be satisfied if and only if ${ }^{31}$

$$
C E(a)= \begin{cases}P E^{+}(a) & \text { if } V(a) R_{i} V\left(a^{\prime}\right) \text { for all } i \text { and } a^{\prime} \in C^{+}(a) \\ P E^{+}(a) \backslash a & \text { if } V\left(a^{\prime}\right) P V(a) \text { for some } i \text { and } a^{\prime} \in C^{+}(a) .\end{cases}
$$

It is straightforward to check if $C E(a)$ is defined above then (CE1), (CE2) and (CE3) are satisfied. So we show the converse.

Consider $C E(a)$ satisfying (CE1), (CE2) and (CE3). The proof proceeds by induction. Note that for any $a \in A^{m}, C E(a)=\{a\}$ and that by the Pareto efficiency of $V$ the claim follows directly. So, consider some $K<m$ and suppose that the claims are true for any agenda in $A^{k}$ for any $k>K$, and let us show that they hold for $a \in A^{K}$.

First, consider the case where $V(a) R_{i} V\left(a^{\prime}\right)$ for all $i$ and $a^{\prime} \in C^{+}(a)$. In this case it follows from (CE2) that $a \in C E(a)$. By the induction step, any $a^{\prime} \in C^{+}(a)$ must result in a Pareto efficient outcome, and so it follows that $a^{\prime}$ is rationalizable relative to $a$ and so by (CE3), $a^{\prime} \in C E(a)$. This implies that $P E^{+}(a) \subset C E(a)$. Also, from the induction step $V\left(a^{\prime}\right)$ is Pareto efficient (relative to $X$ ) for any $a^{\prime} \in C^{+}(a)$, and so $C E(a) \subset P E^{+}(a)$. It follows that $C E(a)=P E^{+}(a)$.

\footnotetext{
${ }^{31}$ Note that in the second case it must be that $P E^{+}(a) \backslash a$ is nonempty.
} 
Next, consider the case where $V\left(a^{\prime}\right) P_{i} V(a)$ for some $i$ and $a^{\prime} \in C^{+}(a)$. In this case, by (CE2) $a \notin C E(a)$. Thus, by nonemptiness (CE1), there is some $a^{\prime} \in C E(a)$, where $a^{\prime} \neq a$. By our induction any $a^{\prime \prime} \in C^{+}(a)$ is Pareto efficient, and so is rationalizable relative to $a^{\prime}$, and so by $(\mathrm{CE} 3) a^{\prime \prime} \in C E(a)$. This implies that $P E^{+}(a) \backslash a \subset C E(a)$. Also, from the induction step $V\left(a^{\prime}\right)$ is Pareto efficient for any $a^{\prime} \in C^{+}(a)$, and so $C E(a) \subset P E^{+}(a) \backslash a$. It follows that $C E(a)=P E^{+}(a) \backslash a$.

Proof of Claim 6: Take any Pareto efficient $x$ such that $x=V(a)$ for some $a=$ $\left(a_{1}, \ldots, a_{m}\right)$. We prove by induction on $k$ that $a \in C E\left(\left(a_{1}, \ldots, a_{k}\right)\right)$ for all $k=1, \ldots . ., m$. The proof is obvious for $k=m$.

Now, assume that the assertion holds for $k>K$ where $K<m$, let us show it holds for $K$. By the Pareto efficiency of $V$, given any $y \neq V(a)$, there exists $i$ such that $V(a) R_{i} y$. By the induction step, $a \in C^{+}\left(\left(a_{1}, \ldots ., a_{K}\right)\right)$, and so (CE3) then directly implies that $a \in C E\left(\left(a_{1}, \ldots ., a_{K}\right)\right)$.

Proof of Theorem 5: We first show the first claim in the theorem. We use Lemma 4. Choose $a \in A$ and any

profile $P \in \mathcal{P}^{*}$. Let $D=\cup_{b \in A^{\text {Full }}(a)} V(b)$. We show that $D$ satisfies the conditions of Lemma 4.

Take any $y \in D$ and $x \notin D$. Since $V$ is Pareto efficient, $y$ is not Pareto dominated by $x$. Given that $P \in \mathcal{P}^{*}$, this means that there is $i \in N$ such that $y P_{i} x$.

Since $D$ satisfies the required condition from Lemma reflem, it follows that $E O(a) \subset$ $\cup_{b \in A(a) \cap A^{m}} V(b)$, as claimed in the theorem.

Next, we show that equality holds if consistency is satisfied. Note that since $V$ is Pareto efficient, the outcomes from full length extensions of $a$ must be Pareto efficient. It follows from Claim 6 that (CE1), (CE2) and (CE3) imply that the equilibrium continuation outcomes following $a$ coincide with the outcomes of full length agenda continuations of $a$.

We now show the claim that the algorithm in Theorem 5 defines a

minimal set of consistent continuation equilibria, even when $V$ may not be Pareto efficient.

Consider the following definition of smallness on continuation equilibria. Given two collections of sets of continuation equilibria $\{C E(a)\}_{a \in A}$ and $\left\{C E^{\prime}(a)\right\}_{a \in A}$, we say that $\{C E(a)\}_{a \in A}$ is smaller than $\left\{C E^{\prime}(a)\right\}_{a \in A}$ if $C E(a) \subset C E^{\prime}(a)$ for all $a \in A$.

The minimal set of continuation equilibria may be identified as follows, as we shall prove below. 
let

We define $C E^{*}(a)$ by induction on the length of $a$. Consider $a \in A$ of length $k$ and

$$
C^{*+}(a)=\cup_{x \notin a} C E^{*}((a, x))
$$

Then we construct $C_{1}^{*}(a)$ as follows.

-Either $V(a) R_{i} V\left(a^{\prime}\right)$ for all $a^{\prime} \in C^{*+}(a)$ and for all $i \in N$. Then $C_{1}^{*}(a)=\{a\}$

-Or $V\left(a^{\prime}\right) P_{i} V(a)$ for some $a^{\prime} \in C^{*+}(a)$ and some $i \in N$. Let $C_{i}(a)$ be the subset of $C^{*+}(a)$ consisting of the agendas $b$ in $C^{*+}(a)$ such that $V(b) R_{i} V\left(a^{\prime}\right)$ for all $a^{\prime} \in C^{*+}(a)$. Then $C_{1}^{*}(a)=\cup_{i \in N} C_{i}(a)$.

Then $C_{2}^{*}(a)$ is defined as the set

$$
\left\{a^{\prime} \in C^{*+}(a): V\left(a^{\prime}\right) R_{i} V(b) \text { for some } b \in C_{1}^{*}(a) \text { and some } i \in N\right\}
$$

Since the set $C^{*+}(a)$ is finite, there exists $j$ such that $C_{j}^{*}(a)=C_{j+1}^{*}(a)$. Define $C E^{*}(a)$ as

such a set.

It follows quite easily from the above construction that $\left\{C E^{*}(a)\right\}_{a \in A}$ is a collection of sets of consistent continuation equilibria.

Next, let $\left\{C E^{* *}(a)\right\}_{a \in A}$ be defined inductively by

$$
C E^{* *}(a)=\left\{\begin{array}{l}
P E^{+}(a) \text { if } V(a) R_{i} V\left(a^{\prime}\right) \forall i \in N \text { and } \forall a^{\prime} \in C^{* *+}(a) \\
P E^{+}(a) \backslash a \text { otherwise }
\end{array}\right.
$$

Claim 18 There exists a unique smallest collection of sets of consistent continuation equilibria which is given by $\left\{C E^{*}(a)\right\}_{a \in A}$ above, and this coincides with $\left\{C E^{* *}(a)\right\}_{a \in A}$.

\section{Proof of Claim 18:}

Step 1: $\left\{C E^{* *}(a)\right\}_{a \in A}$ is a collection of sets of consistent continuation equilibria and $C E^{* *}(a)=C E^{*}(a)$ for all $a \in A$ and all $P \in \mathcal{P}$.

It is straightforward to see that $\left\{C E^{* *}(a)\right\}_{a \in A}$ is a collection of sets of consistent continuation equilibria. We prove the above identity by induction over \#a. Assume that $C E^{* *}(a)=C E^{*}(a)$ for all $a$ such that $\# a>K$ and let $a$ be such that $\# a=K$.

Assume first that $a \in C E^{* *}(a)$. Then it follows from the induction hypothesis that $V(a) R_{i} V\left(a^{\prime}, P\right) \forall i \in N$ and $\forall a^{\prime} \in C^{*+}(a)=C^{* *+}(a)$ and therefore from (CE2), $a \in C E^{*}(a)$. We prove similarly that if $a \in C E^{*}(a)$, then $a \in C E^{* *}(a)$. In that case, if $b \in C E^{* *}(a)$, then $b \in C E^{*}(a)$ as there exists at least one $i \in N$ such that $V(b, P) R_{i} V(a)$. 
Similarly, if $b \in C E^{*}(a)$, then $b \in C E^{* *}(a)$. Assume indeed on the contrary that there exists $c \in C^{*++}(a)$ such that $V(c, P) P_{i} V(b, P) \forall i \in N$. Since from the induction hypothesis, $c \in C^{*+}(a)$, we contradict our construction of $C E^{*}(a)$.

The proof of equality in the case where $a \notin C E^{* *}(a)$

is similar.

Step 2: Step 2: $\left\{C E^{*}(a)\right\}_{a \in A}$ is the unique smallest collection of sets of consistent continuation equilibria.

This follows from Claim 6 and the characterization of $\left\{C E^{*}(a)\right\}_{a \in A}$ in Step 1.

\section{Proof of Proposition 9:}

Both assertions in the proposition follow from Lemma 4 . First, let $D=T C(P)$. Since $T C(P)$ satisfies the requirements of $D$ in the lemma, it follows from that if $V$ is top cycle consistent, then $E O(a) \subset T C(T(P))$.

To prove the second statement concerning Condorcet consistency, let $P$ be any profile with a Condorcet winner, say $x$. Then, let $D=\{x\}$. Since $D$ satisfies the requirements of the lemma, the statement follows.

Proof of Corollary 11: Note the following observations: (i) Any $y$ which beats 0 must have at least $\frac{n+1}{2}$ projects built. (ii) Any $x \in X^{*}(P)$ beats any $y$ such that $y T(P) 0$ and $y \notin X^{*}(P)$ (as then $y$ must involve at least $\frac{n+1}{2}$ projects and yet be more expensive than $x)$.

Using these observations, it follows from (i) than only 0 or some choice of at least $\frac{n+1}{2}$ projects can be the outcome of a full length agenda. From (ii) it follows that only choices in $X^{*}(P)$ can be the outcome of a full length agenda in $A\left(a_{0}\right)$. This implies that only outcomes in $X^{*}(P)$ (if it is nonempty) can be the outcomes of full length agendas in $A\left(a_{0}\right)$. Next note that no element in $X^{*}(P)$ beats any other element in $X^{*}(P)$, and so the first one appearing in the agenda will be the outcome. This means that each element in $X^{*}(P)$ is the outcome of at least one full length agenda in $A\left(a_{0}\right)$. The result then follows from Corollary 10.

Proof of Lemma 14: Let us show that setting $C E(a)=M(a)$ for each $a$ satisfies (CE1)-(CE3).

It follows from the definition of maximal agenda that if $a \in M_{V}(a)$ then $M_{V}(a)=A(a)$ and moreover, that $M_{V}(b)=A(b)$ for all $b \in A(a)$. Then it easily follows that (CE1)(CE3) are satisfied starting at any maximal $a$ at $P$. So, consider $a \in A$ that is not maximal. By the definition of $C E(a)$, it follows that $C^{+}(a)=\cup x \notin a M_{V}((a, x))$. It follows from the definition of maximality that $\cup_{x \notin a} M_{V}((a, x))=M_{V}(a)$. So, $C E(a)=$ $C^{+}(a)=M_{V}(a)$. It then follows directly (noting nonemptiness of $M$ ) that $(\mathrm{CE} 1)$ is 
satisfied. Next, using Pareto efficiency of $V$, since $a \notin M_{V}(a)$, there must be $b \in M_{V}(a)$ such that $V(b, P) P_{i} V(a)$ for some $i$. Since $a \notin M_{V}(a)$, we know that $a \notin C E(a)$, which then satisfies (CE2) since we have established that $V(b, P) P_{i} V(a)$ for some $i$ and $b \in M_{V}(a)=C^{+}(a)$. Finally, note that given Pareto efficiency of $V$, any $b \in M_{V}(a)$ must be Pareto optimal (if not, some $y$ Pareto dominates $x=V(b, P)$, which implies that $y \notin b$; but then by Pareto optimality $V((b, y), P) \neq x$ which is a contradiction). It then follows that for all distinct pairs $b, c \in M_{V}(a)$, there exist $i, j$ with $b R_{i} c$ and $c R_{j} b$. It then follows that all of $C^{+}(a)=M_{V}(a)=C E(a)$ is rationalizable and that (CE3) holds. I

Proof of Theorem 15: Consider an infinite $X$, a Pareto efficient voting rule $V$ and a profile of preferences $P \in \mathcal{P}$ such that $M_{V}(a)$ is nonempty for each $a \in A$.

The remaining part of the proof is identical to that of Theorem 5 after noting that Lemma 4 and Claim 6 remain valid after some slight modification. That is, if $\cup_{b \in A(a) \cap A^{m}} V(b, P)$ is replaced by $\cup_{b \in A(a) \cap M_{V}(a)} V(b, P)$ in Lemma 4, the modified statement remains true. Similarly, Claim 6 can be modified to show that if $V$ is Pareto efficient and $x=V(b, P)$ for some $b \in M_{V}(a)$ for some $a$, then $a \in E O_{V}(a)$. The details are left to the reader. 


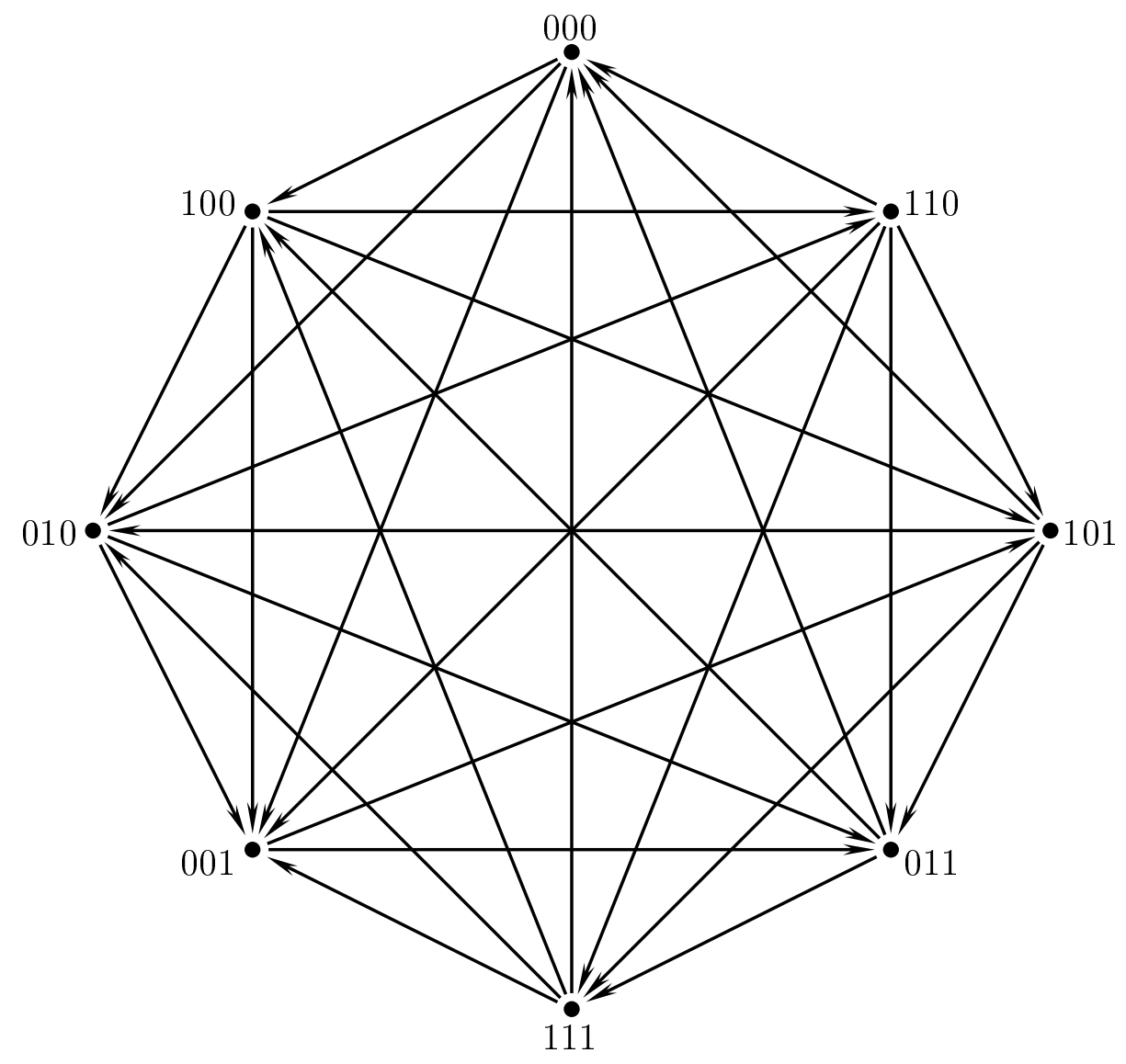

Figure 1: 